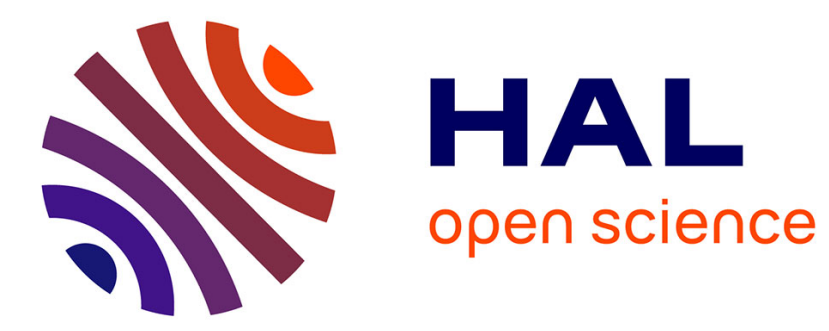

\title{
Large Eddy Simulation of Particle Transport and Deposition Over Multiple 2D Square Obstacles in a Turbulent Boundary Layer
}

\author{
Catherine Le Ribault, I. Vinkovic, S. Simoëns
}

\section{To cite this version:}

Catherine Le Ribault, I. Vinkovic, S. Simoëns. Large Eddy Simulation of Particle Transport and Deposition Over Multiple 2D Square Obstacles in a Turbulent Boundary Layer. Journal of Geophysical Research: Atmospheres, 2021, 126 (16), 10.1029/2020JD034461 . hal-03390550

\author{
HAL Id: hal-03390550 \\ https://hal.science/hal-03390550
}

Submitted on 21 Oct 2021

HAL is a multi-disciplinary open access archive for the deposit and dissemination of scientific research documents, whether they are published or not. The documents may come from teaching and research institutions in France or abroad, or from public or private research centers.
L'archive ouverte pluridisciplinaire $\mathbf{H A L}$, est destinée au dépôt et à la diffusion de documents scientifiques de niveau recherche, publiés ou non, émanant des établissements d'enseignement et de recherche français ou étrangers, des laboratoires publics ou privés. 
Large eddy simulation of particle transport and deposition over multiple $2 \mathrm{D}$ square obstacles in a turbulent boundary layer

\author{
C. Le Ribault ${ }^{1}$, I. Vinkovic ${ }^{1}$, S. Simoëns ${ }^{1}$ \\ ${ }^{1}$ LMFA, ECL, CNRS UMR 5509, UCB Lyon 1 \\ 36, avenue Guy de collongue, F 69134 Ecully Cedex, FRANCE
}

\title{
Key Points:
}

- Large eddy simulation

- Street canyon

- Solid particles 


\begin{abstract}
Predicting solid particle transport in the lowest parts of the atmosphere is a major issue for man-made obstacles in semi-arid regions. Here, we investigate the effects on solid particle saltation, of square obstacles on the ground with different spacings. The aerodynamic field is determined by large eddy simulations coupled with an immersed boundary method for the obstacles. Solid particles are tracked by a Lagrangian approach. Takeoff and rebound models are introduced for the interaction of particles with the wall. Without particles, fluid velocity profiles are first compared with experiments (Simoens et al., 2007) showing good agreement. Special focus is put on the recirculation zone that plays an important role in solid particle entrapment.

Particle concentration fields are presented. Accumulation zones are studied regarding the different obstacle spacings as an extension of the aerodynamic scheme by Oke (1988) to solid particle transport. A deposition peak appears before the first obstacle. When the spacing between the two obstacles is large enough, some particles are trapped within the recirculation and a second deposition peak arises. The streamwise evolution of the horizontal saltation flux shows that the lowest flux downstream of the obstacles is obtained for the highest separation. The deposition rate or the streamwise saltation flux are estimated globally as a function of obstacle spacing. These results illustrate how the numerical tool developed here can be used for assessing air quality in terms of solid particle concentration.
\end{abstract}

\title{
1 Introduction
}

The prediction of solid particle transport, deposition and emission around one or more obstacles, disposed at the ground, is an important issue for cities in the proximity of deserts or in semi-arid regions. Building obstacles is also a widely employed method for stopping or reducing desert progression (Xu et al., 2018). Numerical simulations have widely been used to study natural erodible zones in turbulent boundary layers or above hills (G. Huang et al., 2018, 2019; G. Huang, 2015; Dupont et al., 2013). These simulations can provide detailed information about the evolution of solid particle mass fluxes and help to predict accumulation and erosion zones in a given configuration at different scales. Furthermore, they are independent from a priori global existing laws. Such laws can be used in simple configurations (such as flat terrains) but may be questioned in the case of complex geometries and unsteady flow conditions unlike the present simulation type. Obstacles and buildings greatly modify the characteristics of the incoming boundary layer and induce large recirculation zones where particles are trapped and deposited (G. Huang et al., 2018). In this paper, the influence of squared cross-section obstacles on a flux of eroded sand particles is evaluated. Our goal is to predict preferential deposition or entrainment around square obstacles and to evaluate the influence of the spacing between the obstacles on particle transport and sand fluxes. An attempt is made on proposing global particle transport laws as a function of the roughness parameter for application to larger scale models in view of the law of the wall proposed by G. Huang et al. (2016).

The hopping motion of sand particles, named saltation has largely been studied both through laboratory or in-situ measurements over flat rough surfaces in turbulent boundary layer flows. A large range of empirical models has been developed to describe the physical processes of the interaction between the particles and the ground. Most of these models are summarized by Shao (2008). A first model has been elaborated by Bagnold (1941) to describe the aerodynamic entrainment and to give the threshold velocity at which saltation is initiated. Different analytical threshold velocity estimations have been confronted to wind-tunnel and in-situ measurements since then (Bagnold, 1941; Sørensen, 1991; Diplat \& Dancey, 2013; Foucaut \& Stanislas, 1996). These studies established the parameterization of take-off models for a relatively wide particle size distribution (Descamps 
et al., 2005). Particles in the flow interact with the surface, rebound and some of them eject other particles through the splash process. Rebounding and ejected particles were investigated and modelled through collision experiments between propelling solid particles and a static bed of similar particles (Anderson \& Haff, 1991; Beladjine et al., 2007). In a turbulent boundary layer, in a statistically steady state, the flow eventually reaches an equilibrium due to the negative feedback of the particles on the flow. Models have been developed to take this particle feedback into account by what is called the two-way coupling (Yamamoto et al., 2001; Vinkovic et al., 2006).

Including these type of models, numerical simulations that take into account the complex physical processes of saltation have been developed. The first ones were performed in the idealized case of boundary layers with simple analytical models or presumed behaviour for the velocity field together with a more complete solid particle transport model accounting for ground interactions. Numerical simulations were performed by Kok and Renno (2009), where the wind velocity was estimated through a mean velocity logarithmic profile associated with turbulent velocity fluctuations. This allowed the authors to easily test several physical proposals against experimental results. Parameter variations were then extended to global law applications. From another point of view, a comprehensive numerical model of steady state saltation was developed and used to reproduce a wide range of experimental data. A code based on a Reynolds stress model was developed by Shao and Li (1999) to compute the wind flow and its complete interaction with particles. Simulations were specially focused on the splash process and on the effective roughness length. They achieved to reproduce the experimental streamwise sand drift. This allowed a more detailed and local description of the physical processes involved in sand transport by turbulent flows.

LES has become a well-established tool for the simulation of turbulent flows. This approach allows the computation of the instantaneous evolution of large turbulent structures able to produce sweeping events responsible for the take-off of particles. LES coupled with Lagrangian particle tracking is a particularly suitable approach to simulate solid particle transport. LES were first performed by Vinkovic et al. (2006) to study the dispersion of solid particles in a turbulent boundary layer. It was used to study sand saltation over a flat surface. Later, Dupont et al. (2013) performed large-eddy simulation of turbulent boundary layer flows for different friction velocities and different particle diameters. They showed the existence of aeolian streamers which were then thoroughly inspected. By coupling LES with a discrete element model for tracing medium sand particles, Schmeeckle (2014) conducted eleven numerical simulations to study particle motion over a flat particle bed in the case of sand transport by water. Four-way coupling, meaning two-way coupling together with inter-particle collisions was accounted for. Schmeeckle (2014) studied the evolution of the hydraulic roughness, the rate of shear and bed-load flux. The author found that particle entrainment occurred by fluid penetration of highvorticity turbulence structures referred to as sweeps. More recently, P. Wang et al. (2019) applied large-eddy simulation with particle tracking and two-way coupling to the study of aeolian streamers. By addressing the particle concentration spatial correlation, in the case of a flat particle bed, the authors found that sand streamers exist in the wake of very large turbulent structures.

LES of turbulent dust emissions using a stochastic model were performed by Klose and Shao (2013) to estimate the impact of different thermal stability and wind conditions. Dupont et al. (2014) introduced the influence of vegetation on particle deposition as a first step towards understanding desert progression. Large-eddy simulation performed by ARPS coupled with Lagrangian tracking of solid particles has also been used by N. Huang et al. (2020) to study sand transport under unsteady wind conditions. The simulations showed that sediment transport can be enhanced under unsteady wind and increased with the amplitude of the sinusoidal wind velocity fluctuations. The LES code ARPS coupled with Lagrangian particle tracking has also been applied to the study of snow drift and 
deposition over complex terrain by Z. Wang and Huang (2017). Simulation results showed an overall satisfying agreement with in situ measurements when two-way coupling was accounted for. Updraft on the windward side retarded particle settling while downdraft on the lee side accelerated particle deposition.

Although the Reynolds number is several orders of magnitude lower than in urban canopy atmospheric flows, laboratory experiments provide the controlled conditions necessary for validating saltation models. An experimental campaign was conducted in the frame of the NFSC/ANR sino-french program PEDO-COTESOF "Particle EMission and Deposition Over Complex Terrain for Soil Fixation (PC09)" to investigate particle dispersion over hills (Simoëns et al., 2015), producing both an aerodynamic and solid particle transport description. To evaluate the impact of recirculation zones generated by obstacles on the solid particle mass flux, experiments were performed around one or a set of two $2 D$ Gaussian hills with different spacings by Simoëns et al. (2015). G. Huang et al. $(2018,2019)$ conducted LES of the related cases and compared the results to the experiments by Simoëns et al. (2015). Such LES applications were possible as no a priori mean friction velocity nor global laws depending on friction velocity on flat terrains, were included in the modeling. The flow between and behind the Gaussian hills is dominated by large recirculation zones. The link between these recirculation zones and trapping and erosion was evaluated. The windward side, and the top of the hills were identified as subject to large wind erosion. For the isolated hill case, the windward side was subjected to erosion while particle trapping could potentially occur on the lee side. In the case of double $2 D$ Gaussian hills, potential particle trapping zones were located between the hills. Compared to two successive Gaussian hills, street canyon present abrupt vertical frontiers and corners inducing higher blockage ratios and faster flow deviation with detachment regions. This leads to bigger recirculation regions and the existence of secondary and even tertiary vortices within the canyon. Penetration of the incoming flow within the canyon separation is also reduced when compared to Gaussian hills with similar separations. Therefore, in this study, we will focus on how these specific aerodynamic features of street canyon flows affect particle transport and accumulation.

The configuration of two rectangular cross-section obstacles perpendicular to the mean flow in a turbulent boundary layer represents the synthetic street canyon configuration and has been frequently investigated both experimentally and numerically. Based on momentum and flow mass exchanges between the upper layer and the space between obstacles, Oke (1988) and Grimmond and Oke (1999) identified three basic flow regimes depending on the obstacle separation to obstacle height ratio $R$. Small separation to height ratios $(R \leq 1.5)$ correspond to skimming flow where the canyon contains an isolated vortex with little interaction with the flow above obstacles. For larger ratios, Oke (1988) identified first the wake interference flow regime. In this situation, momentum from the mean flow above the obstacles is directed towards the downstream side of the recirculation region within the canyon, reinforcing it. In this case two counter rotating vortices cohabit inside the cavity. This regime can exist up to roughly a ratio $R$ of 7 . Larger height ratios $(R \geq 10)$ lead to a clear isolated regime where each obstacle behaves as if it was alone. For $7 \leq R \leq 10$ a transitional regime exists where a secondary vortex appears in the windward ground corner of the downstream obstacle. For all these cases a primary vortex is observed in the upstream windward obstacle corner. The length of this vortex is roughly the obstacle height.

Simoens et al. (2007) and Simoëns and Wallace (2008) studied the impact of canyon flows on scalar dispersion by measuring scalar concentration from a two-dimensional source inside the cavity and flushed at the ground at mid-distance between the obstacles. By a kinematic description the authors showed that much of the scalar is trapped between the obstacles. However, rms concentration fields revealed high concentration fluctuations in regions where flow turbulence is rather low. A simple mean concentration gradient model failed in this configuration probably because of rare and intense turbulent events 
above and within the canyon whereas it was successfull in the upper part of the domain where a mixing layer was evidenced. In this study, we are interested in the impact of canyon flows on solid particle transport and specially on saltation and exchanges in the upper part of the cavity. By giving insight into solid particle transport within canyons, this work provides data that can be used for testing large scale time and space averaged prediction models. Moreover, the results presented here can help to elaborate mass flux modeling that accounts for roughness geometrical particularities as it has been done for the law of the wall by G. Huang et al. (2016).

Grigoriadis and Kassinos (2009) studied the effect of the inertial particle response time on the dispersion patterns of a developing flow over an isolated obstacle. Different Stokes numbers, ranging from 0 to 25 were investigated and the effect of particle size was found to have a significant influence on the dispersion pattern over the obstacle. Independently of studies on particle transport over obstacles, straw checkerboard barriers are widely devised for controlling desertification. One or several obstacles are disposed or built on the ground similarly to the street canyon configuration. Hybrid Reynolds averaged Navier Stokes (RANS) / large eddy simulations of saltation over straw checkerboard barriers have been performed by Xu et al. (2018) using Lagrangian particle tracking. The results showed that a majority of particles falls into the checkerboard barrier cells. Checkerboard barrier cells are filled with vortices that transfer sand particles toward the front and side walls. However, the geometry of checkerboard barriers is rather different from street canyon configurations since checkerboards are composed of square cells in the transverse direction. Changes in the geometry imply necessarily differences in the aerodynamic flow features and result in different particle accumulation and deposition patterns.

Although the numerical and experimental investigations cited above have revealed considerable information about street canyon flows, scalar dispersion and particle transport around obstacles, there is still much to be learned. In particular, we provide here a detailed description of the flow, particle concentration and saltation flux within and above the canyon as they vary with canyon openings. Particle saltation is analyzed in view to adapt the flow classification proposed by Oke (1988) to solid particle transport. Contrary to previous studies (Simoens et al., 2007; Simoëns \& Wallace, 2008; Grigoriadis \& Kassinos, 2009), in our simulations, particles are not injected through a slot flushed at the wall but are released upstream the set of two obstacles following an imposed incoming profile of particle concentration induced by well-established saltation laws. The results presented here can be used for testing large scale saltation models that are often based on time averaged mass transport laws and that require particle flux parametrization (Shao \& Leslie, 1997).

The paper is organized as follows. The large-eddy simulation (LES) is described in section 2. Section 3 resumes the model used for the Lagrangian tracking of solid particles in saltation. Main particle-bed interaction models are described in section 4. Simulation parameters are given in section 5 . The validation and discussion of the velocity field is presented in section 6 . Flow velocity profiles are compared to the experimental results (Simoens et al., 2007). Section 7 presents particle concentration, velocity and mass flux results. The influence of obstacle separation on the streamwise sand flux as well as on the deposition or entrainment rates is assessed. Finally, concluding remarks are given in 8 .

\section{Large eddy simulation}

Simulations are performed with the Advanced Regional Prediction System (ARPS version 5.15) code, developed at the university of Oklahoma for predicting atmospheric flows (Xue et al., 1995). The semi-compressible filtered Navier Stokes equations, including momentum, heat (potential temperature), mass (pressure) and the equation of state 
are solved. Subgrid scale turbulence is modeled by a 1.5 order subgrid scale kinetic energy equation (Yoshizawa, 1982).

A fourth-order finite difference method is used for the spatial integration of the equations. The time discretization is performed using a second-order implicit Crank-Nicholson method. The code is parallelized by a domain decomposition method. The full model equations and the numerical method are detailed by Xue et al. (1995, 2000, 2001). Details on the developed extensions for simulating particle transport over obstacles such as $2 D$ Gaussian hills have been described by G. Huang et al. (2019).

Wall-modeling based on the law of the wall is used here. Boundary conditions on the solid wall are imposed through surface momentum fluxes. Grid stretching using a hyperbolic tangent function is applied in the vertical direction. The vertical extent of the domain is divided into three layers. The lowest and the highest layers have a uniform grid spacing. The middle layer has a grid spacing that increases according to the hyperbolic tangent function (G. Huang, 2015). A method for generating three-dimensional, time-dependent turbulent inflow data to simulate complex spatially developing boundary layers is used for the inlet conditions (Lund et al., 1998). The approach is based on extraction/rescaling techniques that produce instantaneous velocity fields from a downstream station far from the inlet.

The ARPS code contains a terrain following model to account for smooth topography changes. This model can not deal with obstacles with vertical walls. An immersed boundary method (IBM) was therefore introduced to account for square obstacles by Le Ribault et al. (2014a). IBM mimics a solid body by a suitably defined body force applied to the discretized set of the momentum equations (Mittal \& Iaccarino, 2005).

On the top of the domain, mirror free-slip boundary conditions are used. Periodic boundary conditions are imposed in the spanwise direction. In the streamwise direction, at the end of the domain, wave-radiation open boundary conditions are used.

\section{Solid particles}

Solid particles are tracked by a Lagrangian approach. Equations for the motion of solid particles have already been presented by Vinkovic et al. (2006). The main features are summarized here.

For solid particles with a diameter $d_{p}$ smaller than the Kolmogorov scale of the fluid flow and a density $\rho_{p}$ much larger than the fluid density $\rho_{f}$, the simplified equations of motion write as:

$$
\begin{gathered}
\frac{d \vec{x}_{p}}{d t}=\vec{U}_{p}(t) \\
\frac{d \vec{U}_{p}}{d t}=\frac{\vec{U}\left(\vec{x}_{p}(t), t\right)-\vec{U}_{p}(t)}{\tau_{p}} f\left(R e_{p}\right)+\vec{g}
\end{gathered}
$$

where $\vec{x}_{p}$ is the particle position, $\vec{U}_{p}$ is the velocity of the particle, $\vec{U}\left(\vec{x}_{p}(t), t\right)$ is the fluid velocity at the particle position and $\vec{g}$ is the acceleration of gravity. The particle relaxation time $\tau_{p}$ is given by:

$$
\tau_{p}=\frac{\rho_{p} d_{p}^{2}}{18 \rho_{f} \nu}
$$

and the particle Reynolds number $R e_{p}$ is:

$$
R e_{p}=\frac{\left|\vec{U}_{p}-\vec{U}\right| d_{p}}{\nu}
$$

where $\nu$ is the fluid viscosity. Particles with a mean diameter of $200 \mu \mathrm{m}$ are studied as described further in section 5. The Kolmogorov length scale estimated in the incoming 
turbulent boundary layer flow according to $\eta \sim \sqrt{\frac{\nu \delta}{0.1 U_{e}}}$ as in Vinkovic et al. (2006) is roughly $\eta \sim 1.5 \mathrm{~mm}$. This confirms the validity of the pointwise particle model within the turbulent boundary layer flow. In the recirculation region between the obstacles, the question of the applicability of the pointwise particle model may arise. However, as in previous studies with particles in recirculation regions we assume that the model is still valid (Escauriaza \& Sotiropoulos, 2011a, 2011b). Effects of nonlinear drag are taken into account by $f\left(R e_{p}\right)$. In this work, an empirical relation is used (Clift et al., 1978):

$$
f\left(R e_{p}\right)= \begin{cases}1+0.15 R e_{p}^{0.687} & \text { if } \operatorname{Re}_{\mathrm{p}}<1000 \\ 0.0183 R e_{p} & \text { otherwise }\end{cases}
$$

The Lagrangian equations and the Eulerian Navier-Stokes equations (computed by the LES) are solved simultaneously. A second order Runge-Kutta scheme is used for time integration of the particle equations. Fluid velocity components resulting from the resolution of the Navier-Stokes equations are only available at discrete mesh nodes. A trilinear scheme of quadratic Lagrange polynomials (Casulli \& Cheng, 1992) is used to interpolate the fluid velocity at the position of the solid particles. The influence of subgridscales on particle transport is not accounted for since the particle to fluid density ratio is large enough and particles are small compared to the smallest turbulent flow scales.

A two-way coupling model is used to account for the influence of the solid phase on the fluid. Small particles, with much larger density than the surrounding fluid act as if they were an extra burden to the fluid and therefore induce a sink of fluid momentum (Elghobashi, 1994). The momentum transfer from particles to fluid is modeled by adding a drag force (two-way coupling) to the fluid momentum equation (Yamamoto et al., 2001). The mean and maximum volume fractions studied here are $\Phi_{p}=5.3 \times 10^{-5}$ and $\Phi_{p, \max }=$ $1.4 \times 10^{-3}$, respectively. The maximum volume fraction is encountered upstream the first obstacle as it will be described further. This value is roughly the upper limit for twoway coupling conditions, enabling us to neglect four-way coupling in this study.

\section{Particle-bed interactions}

Wall-particle interactions are detailed in this section. First the aerodynamic entrainment is presented, then the rebound. Only the main features are recalled here. The models have already been described by G. Huang et al. (2019).

To evaluate the aerodynamic entrainment rate, G. Huang et al. (2018, 2019) developed a new take-off criterion based on the instantaneous evaluation of the different forces exerted on the particle. G. Huang et al. (2019) assumed that take-off occurs when the impulse of the forces acting on the particle (the gravity, the cohesion and the lift forces) at the ground is large enough to disrupt the local equilibrium. Since turbulent structures play a crucial role on the initiation of particle motion (Schmeeckle et al., 2007; Sumer et al., 2003; G. Huang et al., 2019) particle lift is related to strong turbulent sweeps. Contrary to the hill simulations by G. Huang et al. (2019), the present work simulations are performed over a fixed smooth wall. Therefore, here, particles only take-off in areas where they have previously been deposited.

Due to gravity, sand particles fall down and impact the ground. Some of them remain on the ground, others rebound on the soil and can eject several new grains from the bed through the splash process (in the case of available sand particles on the ground). However, in this study, ejection of new grains by splash is not accounted for because only a small number of particles is available on the ground. Once particles deposit on the wall, they can be entrained by aerodynamic forces. However, as described in the next section, we do not start the simulation by aerodynamic entrainment. We rather consider that saltation has already been established upstream the street canyon. Whether a particle 
rebounds or deposits on the ground depends on the velocity and the angle of this particle velocity before the impact, as well as on the characteristics of the soil. Because of its complexity, in this work, rebound is considered as stochastic. Several models have been developed and our approach is mostly derived from the models proposed by Anderson and Haff (1991) and Sørensen (1991). The rebound model has already been described by Dupont et al. (2013). Only the main features are recalled hereafter.

The model is based on the velocity of the impacting particle $v_{i m p}$ and is independent of its diameter (Dupont et al., 2013). The probability $P_{r e b}$ that a particle rebounds when it impacts the surface is given by:

$$
P_{r e b}=0.95\left(1-\exp \left(-\gamma_{r e b} v_{i m p}\right)\right)
$$

where $\gamma_{r e b}$ is an empirical parameter equal to $2 \mathrm{~s} / \mathrm{m}$. The velocity of the particle after the rebound, $v_{r e b}$, is given by a normal distribution:

$$
\operatorname{prob}\left(v_{r e b}\right)=\frac{1}{\sqrt{2 \pi} \sigma_{r e b}} \exp \left(-\frac{\left(v_{r e b}-<v_{r e b}>\right)^{2}}{2 \sigma_{r e b}^{2}}\right)
$$

where $\left\langle v_{r e b}\right\rangle=0.6 v_{i m p}$ is the average of the rebound velocity and $\sigma_{r e b}=0.25 v_{i m p}$ its standard deviation.

The rebound angles toward the surface $\left(\alpha_{v r e b}\right)$ and toward a vertical plane in the streamwise direction of the impacting particle $\left(\alpha_{h r e b}\right)$ are also characterised by a normal distribution with $\left\langle h_{v r e b}\right\rangle=30^{\circ},\left\langle h_{\text {hreb }}\right\rangle=0^{\circ}, \sigma_{v r e b}=15^{\circ}$ and $\sigma_{\text {hreb }}=10^{\circ}$, respectively.

\section{Simulation parameters}

For the present simulations, computational parameters are given in this section. Simulations were performed for an isolated obstacle and for a set of two obstacles with $1 H, 2 H, 4 H$ and $8 H$ spacing, disposed on the wall. The studied geometry is depicted on Figure 1.

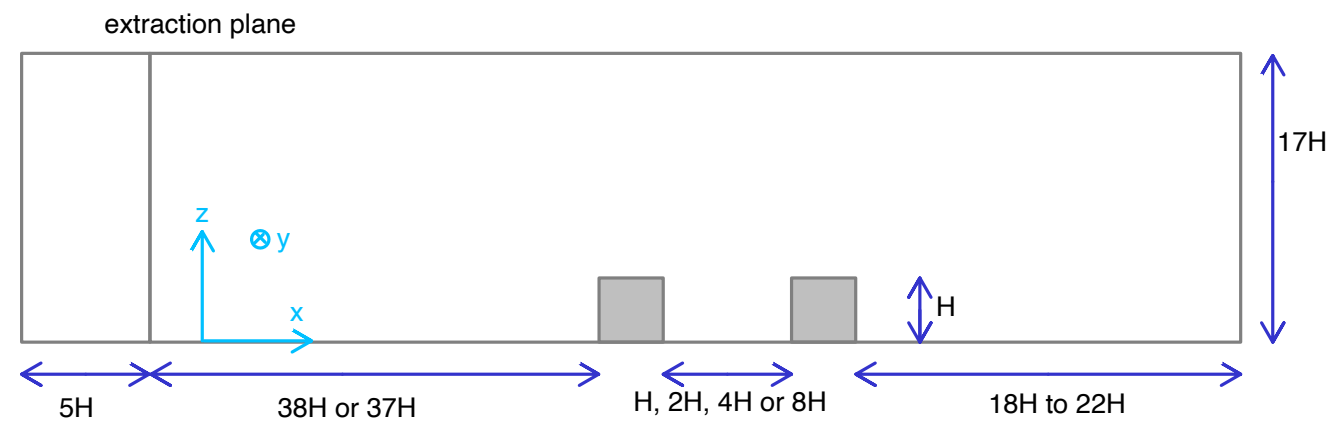

Figure 1. Computational domain (not to scale).

The boundary layer thickness before the obstacles is $100 \mathrm{~mm}$. The square rod obstacles have a cross-section of $10 \mathrm{~mm}$, giving a $1 / 10$ ratio of the obstacle height to boundary layer thickness. The external velocity $U_{e}$ is set to $7 \mathrm{~m} / \mathrm{s}$. Therefore, the corresponding Reynolds number $R e=U_{e} \delta / \nu$ is roughly 46700. The friction Reynolds number is $R e_{\tau}=u_{*} \delta / \nu \sim 2000$ where $u_{*}$ is the friction velocity equal to $0.3 \mathrm{~m} / \mathrm{s}$ at the inlet boundary.

Table 1 summarizes the domain size and the mesh resolution for the computed cases presented here. $L_{x}, L_{y}$ and $L_{z}$ are the sizes of the domain in the streamwise, transverse 
and vertical directions, respectively, as shown on Figure 1. Distances are normalised by the height of the obstacles $H$. For the two obstacle computations with $4 H$ and $8 H$ spacings, the domain is slightly longer $\left(L_{x} / H=70.5\right)$. $L_{x, \text { after }}$ is the size of the domain in the streamwise direction after the second obstacle. $L_{x, o b s}$ is the distance between the inlet and the first obstacle. It is $43 \mathrm{H}$ for the isolated and $1 \mathrm{H}$ separation cases and $42 \mathrm{H}$ for the other two obstacles cases. $\Delta x, \Delta y$ and $\Delta z_{m i n}$ represent the grid steps. The grid is uniform in the horizontal ( $x y$ ) plane and slightly stretched using an hyperbolic tangent function in the vertical direction $(z)$. Computational resolution expressed in wall units are respectively equal to $\Delta x^{+}=23, \Delta y^{+}=46$ and $\Delta z_{\min }^{+}=23$. The problem studied here is statistically homogeneous in the transverse direction. We therefore chose a rather large grid size in this direction. In the streamwise direction a finer grid was needed in order to properly account for the obstacles and the small secondary recirculation zones as it is described further. In the vertical direction, the grid size is the same as in the streamwise direction until $z=1.8 H$, then a stretching is used and the grid becomes coarser.

Results on grid convergence tests are summarized in Appendix A. Moreover, the flow dynamics around an isolated square obstacle have previously been tested in a study dealing with reactive scalar dispersion (Le Ribault et al., 2014b). Tests on different mesh sizes were also performed in the case of one or two consecutive Gaussian hills as described by G. Huang et al. (2018).

Table 1. Grid parameters and domain characteristics

\begin{tabular}{lccccccccc}
\hline Case & $N_{x} \times N_{y} \times N_{z}$ & $\frac{L_{x}}{H}$ & $\frac{L_{y}}{H}$ & $\frac{L_{z}}{H}$ & $\frac{L_{x, \text { obs }}}{H}$ & $\frac{L_{x, \text { after }}}{H}$ & $\frac{\Delta x}{H}$ & $\frac{\Delta y}{H}$ & $\frac{\Delta z_{\text {min }}}{H}$ \\
\hline Isolated & $651 \times 63 \times 90$ & 65.1 & 12.6 & 17 & 43 & 22 & 0.1 & 0.2 & 0.1 \\
\hline $1 H$ & $651 \times 63 \times 90$ & 65.1 & 12.6 & 17 & 43 & 19 & 0.1 & 0.2 & 0.1 \\
\hline $2 H$ & $651 \times 63 \times 90$ & 65.1 & 12.6 & 17 & 42 & 18 & 0.1 & 0.2 & 0.1 \\
\hline $4 H$ & $700 \times 63 \times 90$ & 70.5 & 12.6 & 17 & 42 & 22 & 0.1 & 0.2 & 0.1 \\
\hline $8 H$ & $700 \times 63 \times 90$ & 70.5 & 12.6 & 17 & 42 & 18.5 & 0.1 & 0.2 & 0.1 \\
\hline
\end{tabular}

Solid particles are introduced after mean fluid velocity convergence at $x / H=6$ before the first obstacle. Main solid particle characteristics are given in Table 2. The grains have a mean diameter $d_{p}$ of $200 \mu \mathrm{m}$ with variations between $170 \mu \mathrm{m}$ and $250 \mu \mathrm{m}$ and a density of $1000 \mathrm{~kg} / \mathrm{m}^{3}$.

Table 2. Solid particle characteristics

\begin{tabular}{lcccc}
$d_{p}(\mu \mathrm{m})$ & $\rho_{p}\left(\mathrm{~kg} / \mathrm{m}^{3}\right)$ & $S t_{L}$ & $S t_{\eta}$ & $\gamma_{g}$ \\
\hline 200 & 1000 & 0.84 & 57 & 2.56
\end{tabular}


The global behaviour of particles can be characterized by the Stokes number and the gravity parameter. The Stokes number is defined as the ratio of the particle relaxation time $\tau_{p}$ to a characteristic fluid time and exhibits the ability of the particle to follow the fluid. The Stokes number based on the Lagrangian correlation time scale $S t_{L}$ is equal to 0.84 and the Stokes number based on the Kolmogorov time scale $S t_{\eta}$ is equal to 57. The Kolmogorov time scale has been estimated in the incoming turbulent boundary layer flow according to $\tau_{\eta} \sim \sqrt{\frac{\nu \delta}{\left(0.1 U_{e}\right)^{3}}}$ as in Vinkovic et al. (2006). The gravity parameter $\gamma_{g}$ that gives the ratio between $\tau_{p} g$ ( $g$ being the gravity) and the vertical fluid velocity fluctuations is $\gamma_{g}=2.56$ in this study. This set of Stokes number and gravity parameter indicates that the modified saltation mode is dominant and that the motion of particles is mainly determined by gravity and inertia.

Particles are introduced according to an exponential concentration profile at $x / H=$ 6 before the first obstacle given by Creyssels et al. (2009); Lu et al. (2016):

$$
C(z)=a \exp (-z / b)
$$

with $b=0.025$ as measured by Lu et al. (2016) for $U_{e}=7 \mathrm{~m} / \mathrm{s}$ and $a=1.1$ chosen so that the particle flux $Q$ is in agreement with the empirical prediction of the saltation flux $Q$ (Kawamura, 1951):

$$
Q(x)=2.61 \frac{\rho_{f} u_{*}^{3}}{g}\left(1+\frac{u_{t}}{u_{*}}\right)\left(1-\frac{u_{t}^{2}}{u_{*}^{2}}\right)
$$

where $u_{t}$ is the threshold friction velocity for initiation of saltation.

\section{Fluid velocity validation}

Experiments of flow downstream of an isolated obstacle and across street canyons of variable width disposed along a flat plate were respectively performed by Vinçont et al. (2000), Simoens et al. (2007) and Simoëns and Wallace (2008). The freestream flow speed for these experiments was $U_{e}=2.3 \mathrm{~m} / \mathrm{s}$. The experimental external velocity implies a friction velocity that is below the threshold velocity for saltation for the present sand particles. In our computations, the external velocity has therefore been set to $7 \mathrm{~m} / \mathrm{s}$ in order to obtain saltation. The "Reynolds number independence" hypothesis states that as long as the Reynolds number $\left(R e=U_{e} H / \nu\right)$ is beyond a critical value, the normalized flow field remains invariant with increasing $R e$. In the atmospheric boundary layer, the Reynolds number independence is achieved at roughly $R e \sim 4000$ for a single cube (Castro \& Robins, 1977; Uehara et al., 2003). The criterion Re $~ 10700$ has been adopted for other geometries. Herein, the experimental Reynolds number is 10000, whereas the Reynolds number of the simulation is $R e \approx 46700$. In so dynamically, the experiments (Vinçont et al., 2000; Simoens et al., 2007; Simoëns \& Wallace, 2008) may be used to validate the flow dynamics.

\subsection{Recirculation zone}

The recirculation zones obtained for the different configurations are presented in Figure 2. All lengths have been normalized by the height of the obstacles $H$. In the case of the isolated obstacle, the $x$-axis origin is set at the upstream face of the first obstacle. For the set of two obstacles, the origin is set at the mid-distance between the two obstacles.

For all cases, a primary vortex, with negative spanwise vorticity, forms within the canyon and is driven by the flow above (Figure 2). For the isolated obstacle, the length of this recirculation zone is roughly $7 \mathrm{H}$. A secondary recirculation zone of approximately 

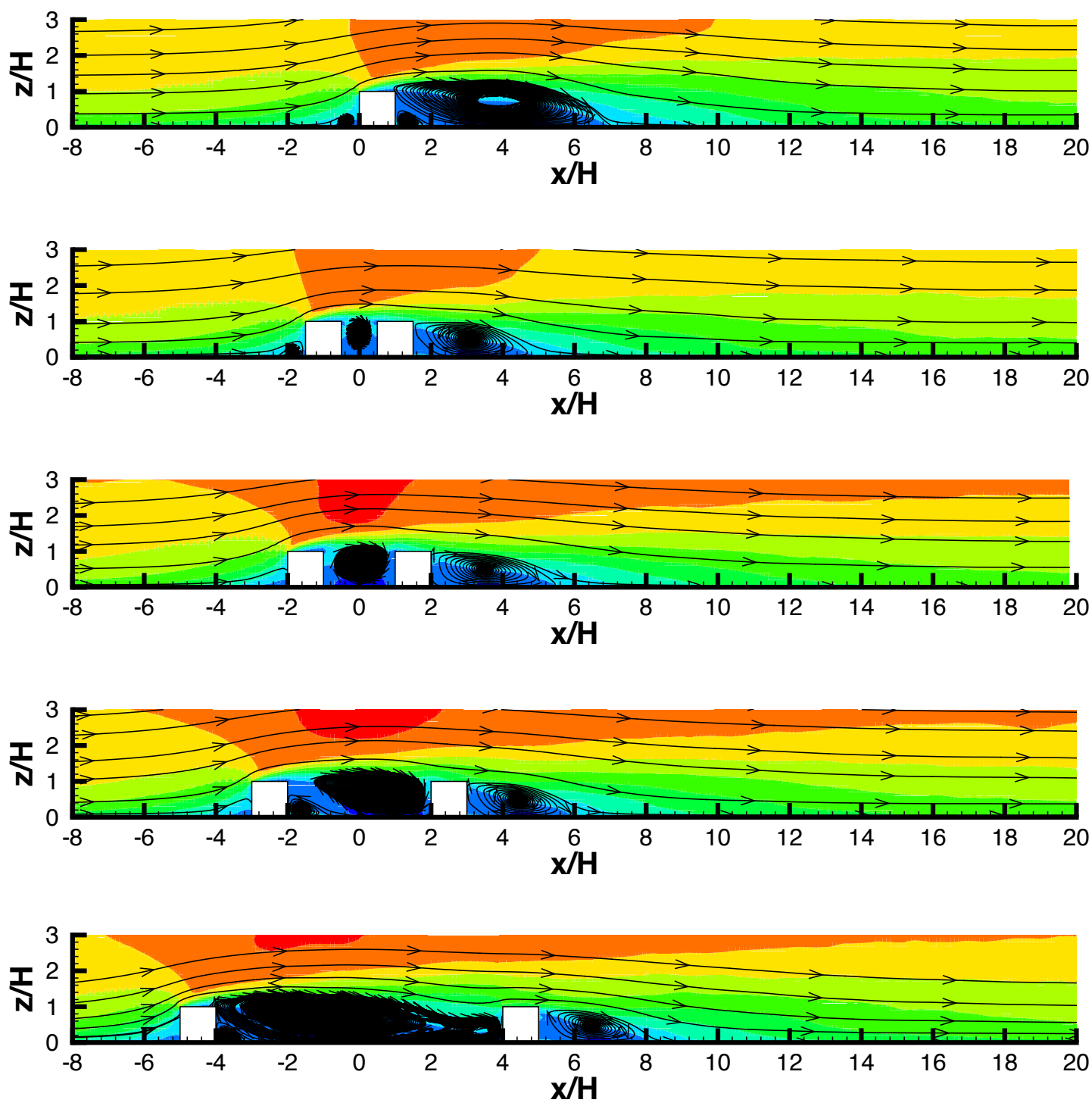

Figure 2. Recirculation zones for the isolated obstacle and for the obstacles with $1 H, 2 H, 4 H$ and $8 H$ spacing (from top to bottom). Colored isocontours give the mean streamwise velocity $U$ in $\mathrm{m} / \mathrm{s}$. Streamlines are superposed.

$H$ appears in the corner at the leeside downstream the isolated obstacle. This pattern is in agreement with the experimental observations by Vinçont et al. (2000) and has also been obtained by Grigoriadis and Kassinos (2009) from LES computations.

In the case of two consecutive obstacles, for the openings of $1 H$ and $2 H$, the center of the primary recirculation zone is roughly centered in the middle of the canyon. For the $4 H$ case, the center of the vortex slightly shifts downstream of $x / H=0$. For wider obstacle separations $(8 H)$, the primary vortex core center slides upstream of the middle of the canyon becoming closer to the upstream obstacle. For all obstacle separations, recirculation zone streamlines spread above the top of the obstacles suggesting that the primary vortex extends above the level of the buildings. These numerical findings confirm previous experimental observations by Simoens et al. (2007). 
For $1 H$ and $2 H$ obstacle separations, no secondary vortex with positive spanwise vorticity is observed within the canyon. This secondary recirculation appears in the upstream corner of the canyon when the separation is increased to $4 H$ and further. Experimentally, Simoens et al. (2007) captured the presence of this secondary corner vortex for a $2 H$ opening. Moreover, experiments performed by Sato et al. (2015) with PIV measurements show the existence of such a vortex for a $3 H$ spacing. Such a vortex is symptomatic of an instability induced at the top of the cavity. It is parameterized by the cavity width $(C W)$. The threshold width $C W_{t}$ passing the vortex number from one to two inside the cavity is around $1.5<C W<2.5$, in so any disturbance can switch on the appearance of this second vortex. In our simulations, a precise prediction of corner flows is constrained by the difficulty of the near wall modelling and the coupling with the immersed boundary method. Meshes representing near wall corners cumulate numerical difficulties related to both methods, namely wall modelling and IBM.

For $8 H$, three recirculation zones are present within the canyon. A small secondary vortex of $1 H$ is located in the corner of the first obstacle. A big primary recirculation zone of $7 \mathrm{H}$ appears upstream of the middle of the canyon. The length of the big vortex is approximately equal to the size of the recirculation zone after the isolated obstacle $(7 H)$. Finally a tertiary vortex with the same negative spanwise vorticity as the primary vortex appears at the downstream corner of the cavity. For the widest opening $(8 H)$, the large recirculation zone reattaches and the flow within the cavity begins to re-establish itself as a boundary layer before reaching the downstream obstacle. This pattern shows that the canyon between the two obstacles is large enough so that the interdependence of consecutive obstacles weakens. This separation could be qualified as the beginning of the 'isolated flow' regime according to classification of Oke (1988). As such regime seems transitional till $10 \mathrm{H}$ spacing, we call it transitional isolated regime in the rest of the text.

For all cases, a small vortex appears upstream the first obstacle. In the experiments (Simoens et al., 2007), the size of this vortex is approximately equal to the size of the obstacle. In the simulations it is slightly smaller and this difference may come from the coupling between the law of the wall used to compute the flow in the near-wall region and the IBM applied for the obstacles which are less accurate in corners and junctions between walls and obstacles.

For all cases, the simulated velocity patterns are in overall agreement with the experimental observations (Vinçont et al., 2000; Simoens et al., 2007; Simoëns \& Wallace, 2008) although the Reynolds number differs. Globally, the mean flow behaviour over the two squared obstacles with different spacings can be characterized according to the classification given by Oke (1988). Spacings of $H$ and $2 H$ fall within the skimming flow regime since only one primary recirculation zone is observed. The $4 H$ opening corresponds to wake flow where a small part of the incoming flow penetrates the canyon, reinforcing the primary recirculation zone and creating a secondary vortex with negative vorticity in the upstream corner within the canyon. Finally, from this qualitative analysis of flow patterns, transitional isolated flow is obtained by the $8 H$ spacing where downstream the primary recirculation region the boundary layer flow is briefly re-established before reaching the downstream building. We qualified the $8 H$ separation as a transitional isolated case and not as a fully isolated case since the two recirculation zones observed in the canyon interact.

\subsection{Mean velocity}

The mean streamwise velocity $U$ profiles are presented in Figure 3 for the different configurations. The average streamwise velocity is normalised by the external velocity $U_{e}$. The experimental profiles of Simoens et al. (2007) are added for validation and comparison. Experimental error bars of roughly $7 \%$ of the external velocity have been added to the measured values. The experimental error is mostly due to rather low PIV 

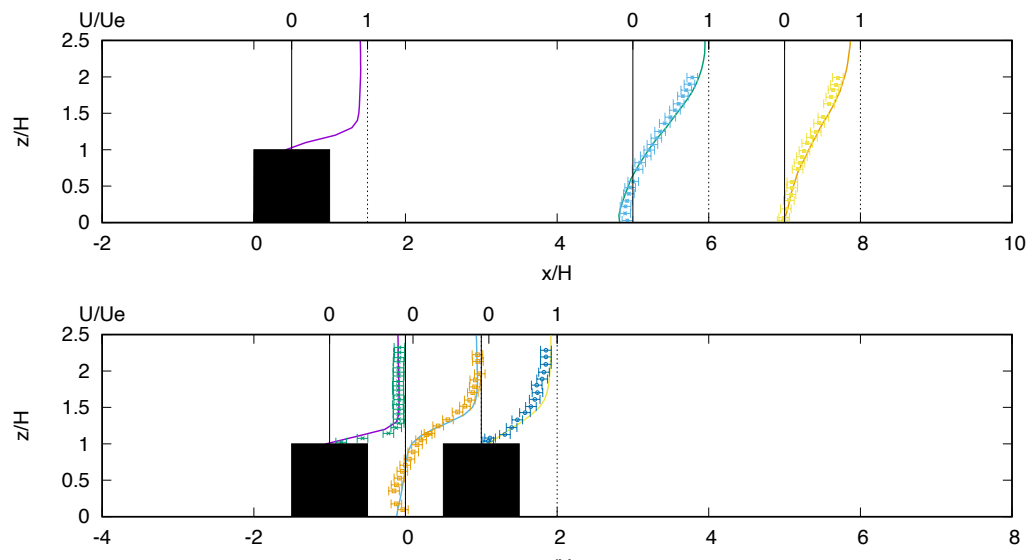

$\mathrm{x} / \mathrm{H}$
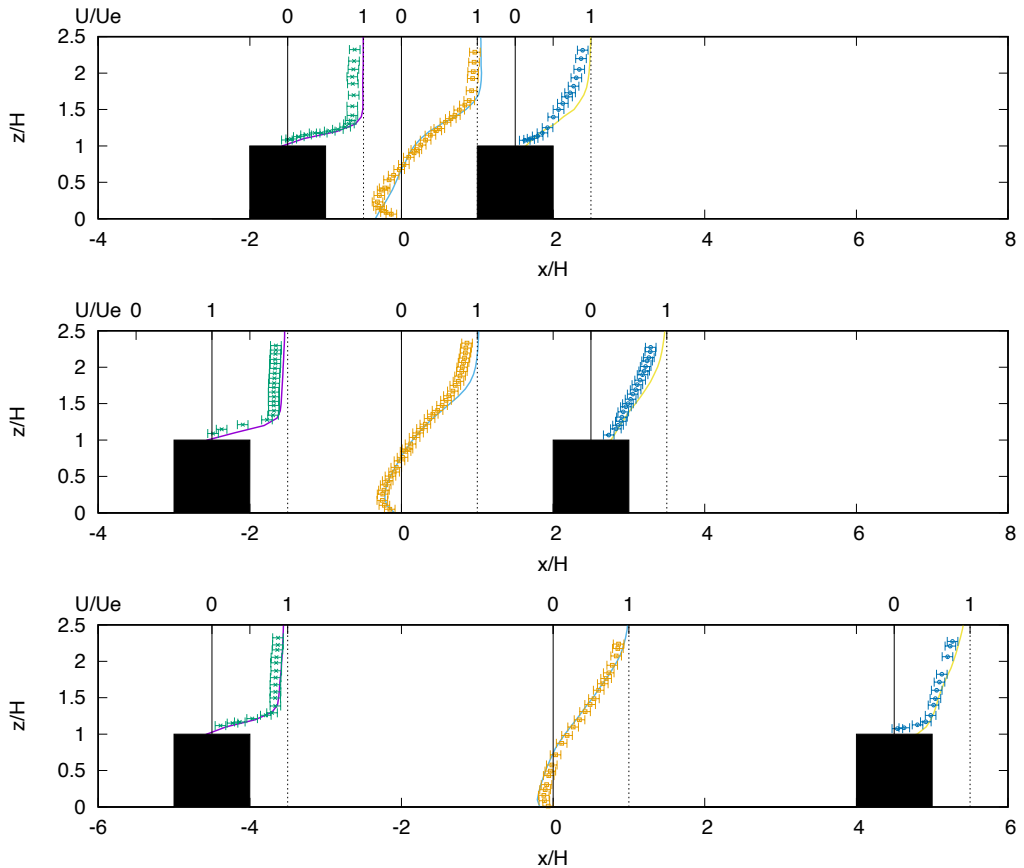

Figure 3. Mean streamwise velocity profiles for the isolated obstacle and for the $1 H, 2 H, 4 H$ and $8 H$ (from top to bottom) canyon openings. Lines - LES. Symbols - experiments (Simoens et al., 2007).

(particle image velocimetry) resolution in the near wall region of the flow, implying large bias on low velocity measurements.

For the isolated case, the profiles are presented above the obstacle and at $5 H$ and $7 \mathrm{H}$ after the obstacle, where experimental profiles are available. Mostly, numerical profiles fall within the error bar interval of the experimental results. Therefore, LES results are in good agreement with the experiments confirming a self-similarity for this Reynolds number range. At $5 H$, near the center of the recirculation zone as shown on Figure 2, the near wall velocity is negative. At $7 H$ and further downstream, the flow recovers the boundary layer shape.

For the two obstacle cases, profiles are plotted at the middle of the first obstacle, between the two obstacles and at the middle of the second obstacle as experimental re- 
sults are also available at these sections. Above the upstream obstacle, the velocity profiles are similar for the different cases and in agreement with experimental results.

Within the canyons, at the ground, for all the cases, the mean streamwise velocity is negative due to the recirculation zone. The experimental velocity is equal to zero at the ground and the maximum is slightly above the wall. In the simulation, the velocity at the wall is not equal to zero, due to the law of the wall imposing that the first simulation node is located within the logarithmic layer. The region of negative mean streamwise velocity extends from the wall to roughly $0.8 H$. The largest negative mean streamwise velocity is obtained for the $2 H$ separation case. Both the maximum and the height of the negative velocity close to the wall in the middle of the canyon are well predicted and in good agreement with the experiments (Simoens et al., 2007). Small discrepancies appear for $1 H$ and $2 H$ canyon openings probably due to a small lag in the center of the recirculation zone. It is rather difficult to accurately predict the exact position of the recirculation region or of two consecutive recirculation regions in the case of canyon openings larger than $2 H$. A small under- or overestimation of the addressed recirculation regions lead to rather important differences in the average velocity profiles. Namely, both for the experiments as for the simulations, velocity profiles are strongly influenced by vertical gradients found within recirculation zones implying greater differences inside than outside recirculations.

Above the canyon, the mean streamwise velocity profiles indicate that the vertical extent of the shear layer increases with obstacle separation even though the magnitude of mean shear slightly decreases.

Above the downstream obstacle, numerical simulations predict rather well the mean flow velocity experimental profiles. There is a slight overestimation of the simulated mean velocity above $z / H>1.5$ for the two smaller spacings. In the case of $4 H$ and $8 H$ spacing an overall satisfactory agreement is achieved. With increasing obstacle separations, mean streamwise velocity profiles above the downstream obstacle become less rounded. Experiments (Simoens et al., 2007) suggested the existence of a thin region of recirculation flow with small negative streamwise velocity just above the top of the upstream and the downstream obstacles for $8 H$ separations. Unfortunately, this has not been captured by our LES probably due to the precision of the interpolation scheme used in the immersed boundary method just above the roof top ( $\mathrm{Wu}, 2019)$.

\subsection{RMS velocity}

The mean Reynolds stress denoted here $u^{\prime} w^{\prime}$ and normalized by $U_{e}^{2}$ is presented on Figure 4 for the five different configurations. For all cases, a peak of negative $u^{\prime} w^{\prime}$ appears above the first obstacle. After the first obstacle, a peak of positive $u^{\prime} w^{\prime}$ appears at the same height, roughly $1.2 H$ above the wall. The shear layer spreads in the longitudinal direction and the peak decreases in intensity. For the $1 H$ and $2 H$ configurations, the shear layer stays above the set of the two obstacles. For the $4 H$ and $8 H$ configurations, high levels of $u^{\prime} w^{\prime}$ penetrate inside the canyon. The spread of the high intensity $u^{\prime} w^{\prime}$ layer is shifted upwards by the second downstream obstacle. Further downstream, this $u^{\prime} w^{\prime}$ layer spread decreases in intensity. It eventually reaches the wall at roughly $2 H$ after the second obstacle. Perturbations of the incoming flow by the presence of obstacles are observed in terms of $u^{\prime} w^{\prime}$ very far away from the last obstacle. They may reach as far as $15 H$ for the isolated case and roughly $13 H$ for the double obstacle cases. Slight $u^{\prime} w^{\prime}$ numerical oscillations are observed upstream the first corner of the first obstacle. These oscillations are produced by the coupling of the IBM with the LES on singularities such as the corner. They can be suppressed by much refined meshes together with filtering procedures as described by Uhlmann (2005).

The streamwise RMS velocity noted $u^{\prime}$ for the different configurations is presented in Figure 5 and results are compared to experimental profiles for the same sections as 

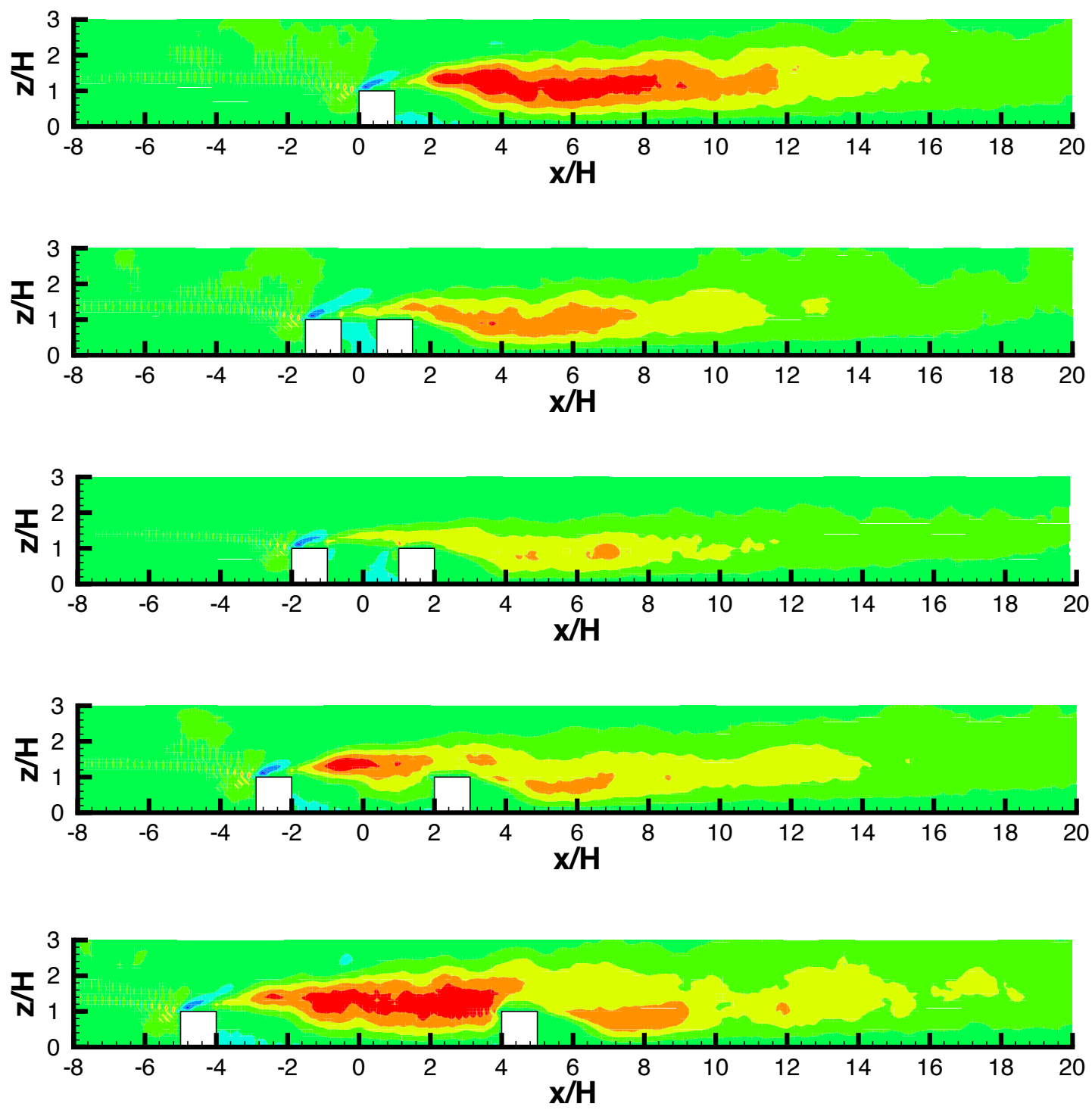

Figure 4. Reynolds stress isovalues $u^{\prime} w^{\prime}$ for the isolated obstacle and for the obstacles with $1 H, 2 H, 4 H$ and $8 H$ spacing (from top to bottom). Values are normalized by $U_{e}^{2}$.

for the average velocity (Figure 3). Experimental error bars of roughly $10 \%$ of the maximum $u^{\prime}$ value have been added.

For the isolated obstacle, at the first section above the obstacle, the peak of RMS velocity appears at $0.2 \mathrm{H}$ above the obstacle. At $5 \mathrm{H}$ and $7 \mathrm{H}$, the peak remains at the same height of roughly $1.2 \mathrm{H}$ above the wall even though it is noticeably spread and diminished. For each section, the maximum is located near the inflection point of the corresponding mean velocity profile, at the position of the highest mean velocity gradient.

For the two obstacle cases, a narrow high intensity layer of turbulence of about $20 \%$ of the maximum mean velocity appears above the upstream obstacle for all canyon openings. The peak is approximately located at $1.2 \mathrm{H}$ above the wall, as for the isolated case. 

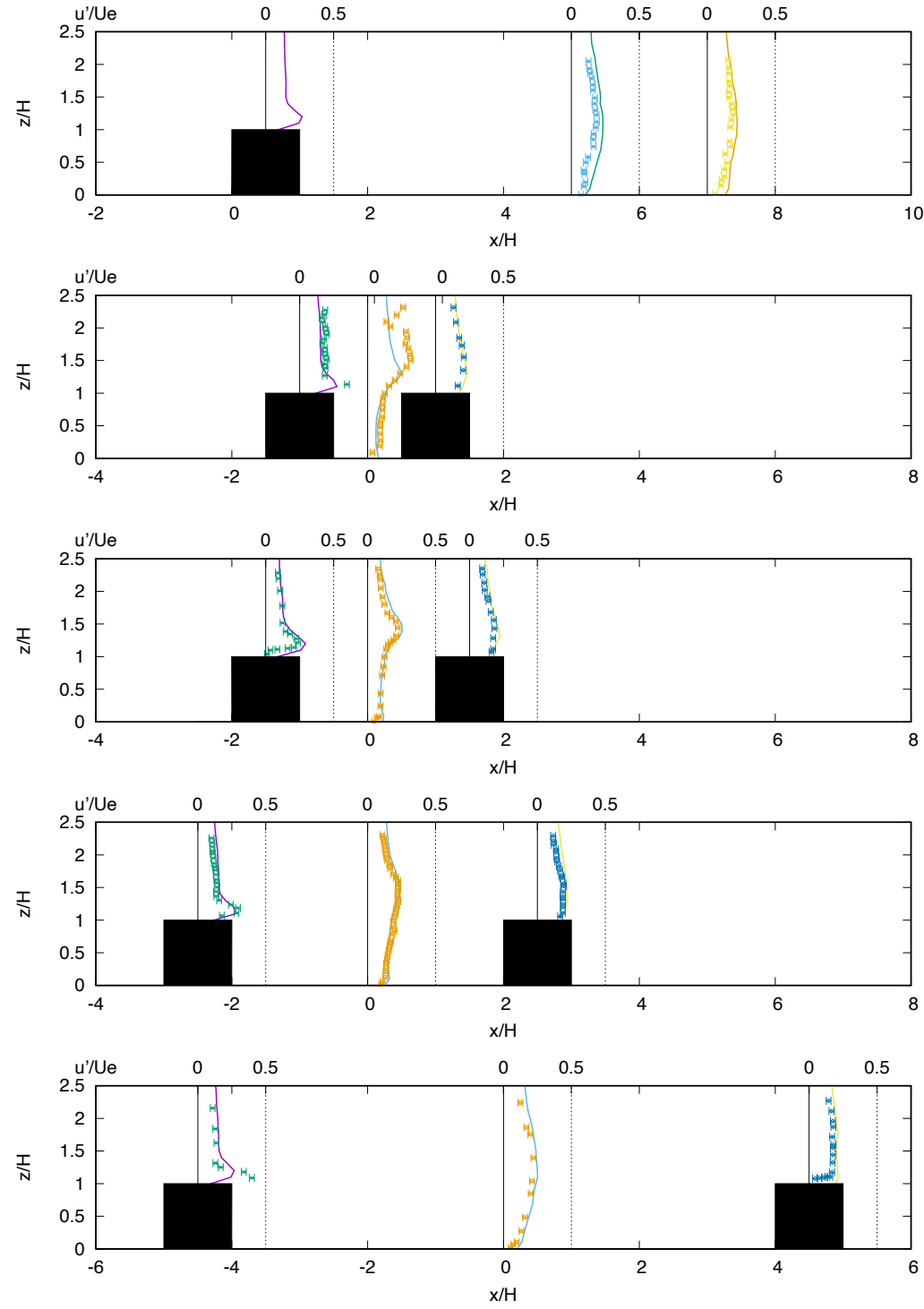

Figure 5. RMS streamwise velocity fluctuation profiles for an isolated obstacle and for the two obstacles cases with $1 H, 2 H, 4 H$ and $8 H$ distances (from top to bottom). Lines - LES. Symbols - experiments (Simoens et al., 2007). 
For $1 H$ and $2 H$ obstacle separations, the maximum peak above the canyon slightly decreases in intensity and rises due to the spreading of the shear layer above the top of the obstacle on the upper vertical limit of the shear layer. This suggests that high velocity fluctuations spread away from the canyon depicting the skimming flow regime in the classification of Oke (1988) where the incoming flow perturbations fail to penetrate the canyon.

For $4 H$ and wider canyon openings, the streamwise velocity rms profiles at $x / H=$ 0 are flattened and higher levels of flow velocity fluctuations penetrate the canyon. As the obstacle separation is increased to $4 H$ and further, the velocity fluctuations penetration spreads filling vertically the canyon with lower fluctuation levels. This is qualitatively in accordance with the wake and isolated flow regimes (Oke, 1988) where the incoming perturbations reach the canyon gap and influence the flow between the obstacles.

Above the downstream obstacle, the intensity of the streamwise fluctuations is reduced as the canyon opening increases. An overall satisfying agreement is achieved between the LES and the experimental results by Simoens et al. (2007) for the isolated as well as for the two obstacle cases with different spacings. Discrepancies with the experimental values appear above the first obstacle for the $8 H$ canyon opening pointing the difficulty of accurately predicting near wall flows by LES coupled with wall modelling and an immersed boundary method.

\subsection{Friction velocity}

To investigate the link between particle deposition and the friction velocity, local time averaged (LTA) and spatial and time averaged (STA) friction velocities $u_{*}$ and $u_{*}^{m}$ around the obstacles are presented and discussed as in G. Huang et al. (2018). The averaged shear velocities are often applied in models for the entrainment of solid particles and as threshold values for the initiation of sand particle transport (Shao, 2008). Figure 6 shows the local time averaged friction velocity $u_{*}=\sqrt{\left|u^{\prime} w^{\prime}\right|}$ scaled by the inlet shear velocity $u_{*}^{0}$ for the five cases studied here. In reversal flow parts, $u_{*}$ is taken as negative as previously used for Gaussian hills by G. Huang et al. (2018).

Before the first obstacle, the friction velocity $u_{*}$ has the same behaviour in the five configurations. It stays approximately constant until the small upstream vortex before the first obstacle and then decreases abruptly close to the obstacle. Surprisingly, the intensity of the decrease is stronger for the $2 H$ obstacle separation. For this separation, the incoming flow is further perturbed by the presence of the obstacles than in the other cases. In the skimming flow case, since the incoming flow cannot penetrate the canyon, the canyon appears as one big obstacle to the flow. Changes in the incoming flow are therefore even more pronounced in the case of this skimming flow regime. This might have an important impact on particle saltation.

After the first obstacle, the friction velocity depends on the configuration of the recirculation bubbles. For the isolated obstacle, it remains positive into the first small recirculation zone after the obstacle and then becomes negative and decreases until the end of the big primary recirculation bubble. After the reattachment point, it increases abruptly to reach a constant value slightly higher than the incoming one $\left(u_{*}^{0}\right)$ at about $7 H$ after the obstacle.

For the $H$ and $2 H$ cases, the friction velocity is negative between the two obstacles and tends to zero in the center of the vortex. After the second obstacle, the friction velocity is still negative in the reversal flow. A similar behaviour of the time averaged friction velocity obtained for $H$ and $2 H$ separations may confirm that these two cases belong to the same skimming flow regime where only one primary recirculation zone is observed centered on the middle of the canyon. 

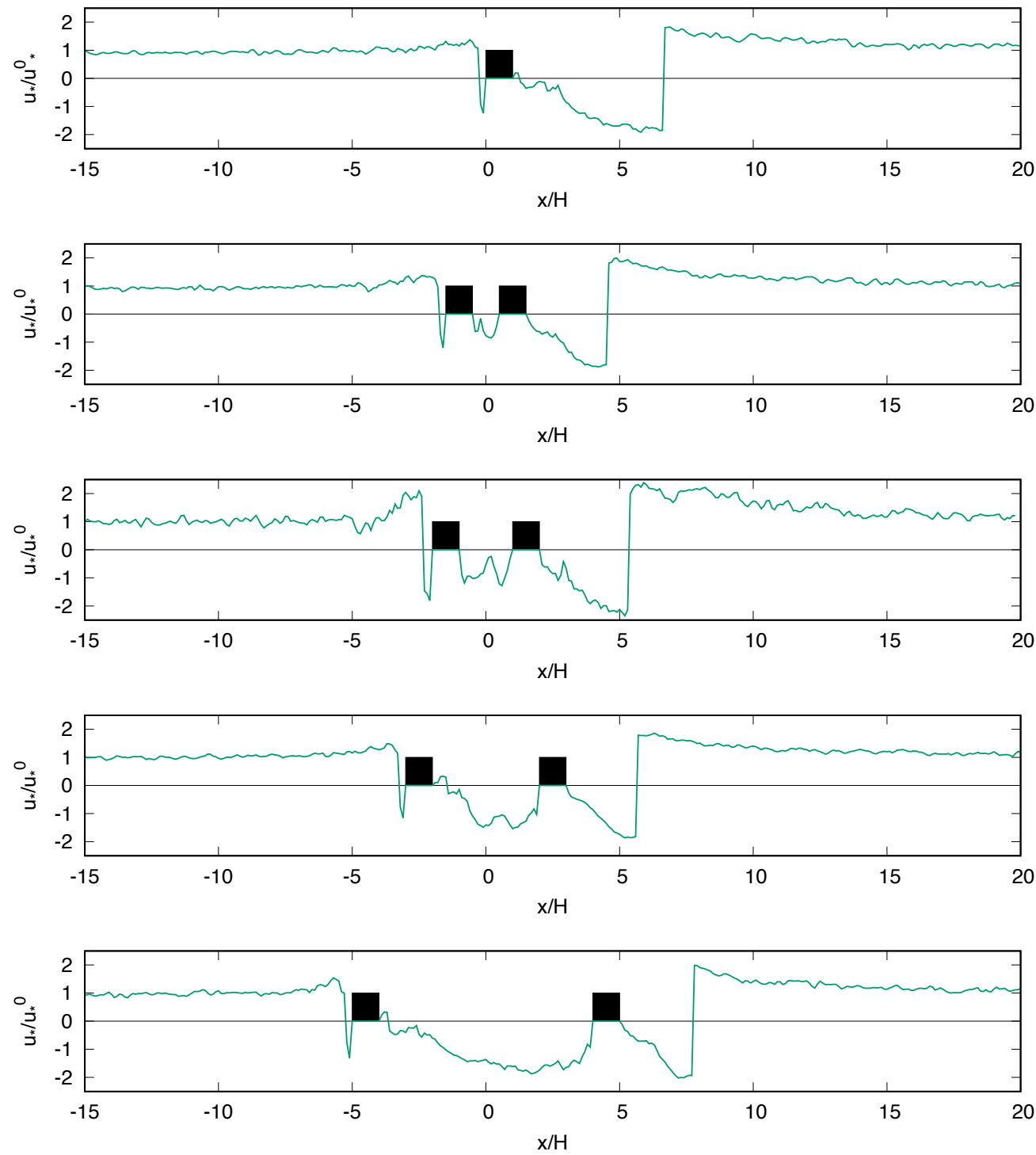

Figure 6. Local time averaged friction velocity for an isolated obstacle and for the two obstacles cases with $1 H, 2 H, 4 H$ and $8 H$ distances (from top to bottom). 
For the set of obstacles separated by $4 H$ and $8 H$, three recirculation zones are present between the two obstacles. The friction velocity is positive in the small first recirculation zone after the first obstacle and then remains negative in the reversal flow. For the $8 H$ opening, the separation between the primary and the tertiary recirculation bubbles is captured by the slight increase of $u_{*}$ at roughly $x / H \sim-3$. The streamwise evolution of the friction velocity of the $8 H$ separation case does not present the abrupt increase at the end of the primary recirculation zone as in the isolated case. Moreover, the evolution of $u_{*}$ for the $8 H$ opening is qualitatively closer to the behaviour of the wake flow regime ( $4 \mathrm{H}$ separation). The largest opening studied here $(8 H)$ does not fall fully under the isolated flow regime. Since it presents a tertiary negative vorticity recirculation region, without a net reattachment of the primary recirculation, the $8 H$ separation case is rather set on the limit between the wake flow and the isolated flow regime.

In regions where the friction velocity is positive (upstream of the obstacles), particle transport is carried on along the prevailing wind direction of the upper flow. Within the recirculation zone, since the flow changes its direction near the wall, backflow transports particles towards the lee side of the obstacles. This reverse transport is purportedly the origin of particle trapping within the recirculation zone (Araújo et al., 2013) and therefore within the canyon intergap. The presence of large regions of negative friction velocity may induce the presence of large particle deposition areas behind or between the obstacles. This gives a clue on how obstacles might be used to stabilize bed erosion or how air quality may be highly altered close to the ground within street canyons.

Meso-scale global particle transport models often use not only time-averaged values of the friction velocity but also space averages over subgrid scales reaching several meters (Shao \& Leslie, 1997). It is therefore also interesting to compute the mean along the streamwise $x$ axis values of $u_{*}^{m} / u_{*}^{0}$. This characterizes the global friction for the different cases computed here from a mesoscale point of view and gives parameter trends for cases of fields covered with such patterns without detailing them.

The LTA normalized absolute value of the friction velocity $\left|u_{*}\right| / u_{*}^{0}$ is longitudinally averaged on a domain for which the turbulent boundary layer is modified by the obstacles. The average is performed between $x_{1}$ and $x_{2} . x_{1}$ is chosen at $-2 H$ before the first obstacle and $x_{2}$ is set at the end of the recirculation zone after the second obstacle, where the friction velocity reaches a constant value. This space and time mean friction velocity averaged between $x_{1}$ and $x_{2}$ is denoted by $u_{*}^{m}$. Another averaging is performed only within the canyon in two obstacles cases. This other average friction velocity is denoted by $u_{*}^{m b}$ and is also normalized by the inlet friction velocity.

The obtained $u_{*}^{m} / u_{*}^{0}$ and $u_{*}^{m b} / u_{*}^{0}$ values for different obstacle separations as well as the values of $x_{1}$ and $x_{2}$ are given in Table 3 . Figure 7 shows the evolution with street canyon opening of the STA friction velocity $u_{*}^{m} / u_{*}^{0}$ averaged between $x_{1}$ and $x_{2}$ and of $u_{*}^{m b} / u_{*}^{0}$ averaged only within the canyon.

For small canyon openings $(1 H$ and $2 H)$, the STA friction velocity $u_{*}^{m}$ increases compared to the inlet value when it is averaged between $x_{1}$ and $x_{2}$. For this averaging procedure, the highest value of $u_{*}^{m} / u_{*}^{0}$ is obtained for the $2 H$ separation corresponding to skimming flow. The averaged ratio $u_{*}^{m} / u_{*}^{0}$ between $x_{1}$ and $x_{2}$ then drops slightly below the isolated case level for the two largest street canyon openings $(4 H$ and $8 H)$. When the flow before the first obstacle is accounted for in the averaging procedure, the STA friction velocity is the highest in the skimming flow case. This corresponds to the earlier stated description of a skimming flow that sees the two consecutive obstacles as one big obstacle since the incoming flow does not penetrate into the cavity. For the two obstacle configurations, when $\left|u_{*}\right| / u_{*}^{0}$ is averaged only within the canyon, the STA friction velocity $u_{*}^{m b} / u_{*}^{0}$ decreases compared to the isolated obstacle. The value is minimum for the smallest separation. The overall friction is clearly reduced within the canyon. The reduction is stronger when the separation is smaller. If we only look at what happens 


\begin{tabular}{cccccc}
\hline & Isolated & $\mathrm{H}$ & $2 \mathrm{H}$ & $4 \mathrm{H}$ & $8 \mathrm{H}$ \\
\hline$x_{1}$ & -2 & -3.5 & -3 & -4 & -6 \\
\hline$x_{2}$ & 8 & 6 & 6 & 7 & 8 \\
\hline$u_{*}^{m} / u_{*}^{0}$ & 1.22 & 1.27 & 1.44 & 1.17 & 1.20 \\
\hline$u_{*}^{m b} / u_{*}^{0}$ & 1.22 & 0.62 & 0.82 & 0.92 & 1.17 \\
\hline
\end{tabular}

Table 3. STA friction velocity $u_{*}^{m} / u_{*}^{0}$ averaged between $x_{1}$ and $x_{2}$ and $u_{*}^{m b} / u_{*}^{0}$ averaged only within the canyon obtained by LES

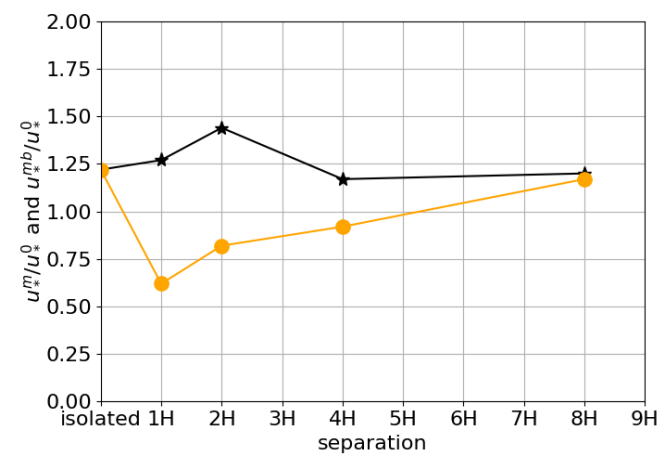

Figure 7. Local time and space averaged friction velocity $u_{*}^{m} / u_{*}^{0}$ evolution with obstacle separation. Stars $-u_{*}^{m} / u_{*}^{0}$ averaged between $x_{1}$ and $x_{2}$. Points $-u_{*}^{m b} / u_{*}^{0}$ averaged only within the canyon.

within the canyon $\left(u_{*}^{m b} / u_{*}^{0}\right)$, the strongest friction reduction is obtained for the skimming flow, once again because for small separations the flow within the canyon is sheltered from the incoming high velocity fluid. This decrease in the time and space average of the friction velocity within the canyon, in the presence of obstacles, gives an indication of the overall reduction of flow friction in the presence of built objects. It also illustrates that the presence of obstacles induces flow patterns that are suitable for particle deposition and entrapment. The lower the average space and time friction within the canyon, the more particle deposition there might be. Therefore, two obstacle configurations provide an overall shelter for solid particle transport by shattering friction. Values of the STA friction velocity given in Table 3 can be used as boundary conditions in meso-scale simulations.

\section{Particle saltation around obstacles}

Results related to solid particle transport are presented here. First, concentration and particle velocity profiles are discussed. Then, results on particle deposition, emission and saltation fluxes are presented. 

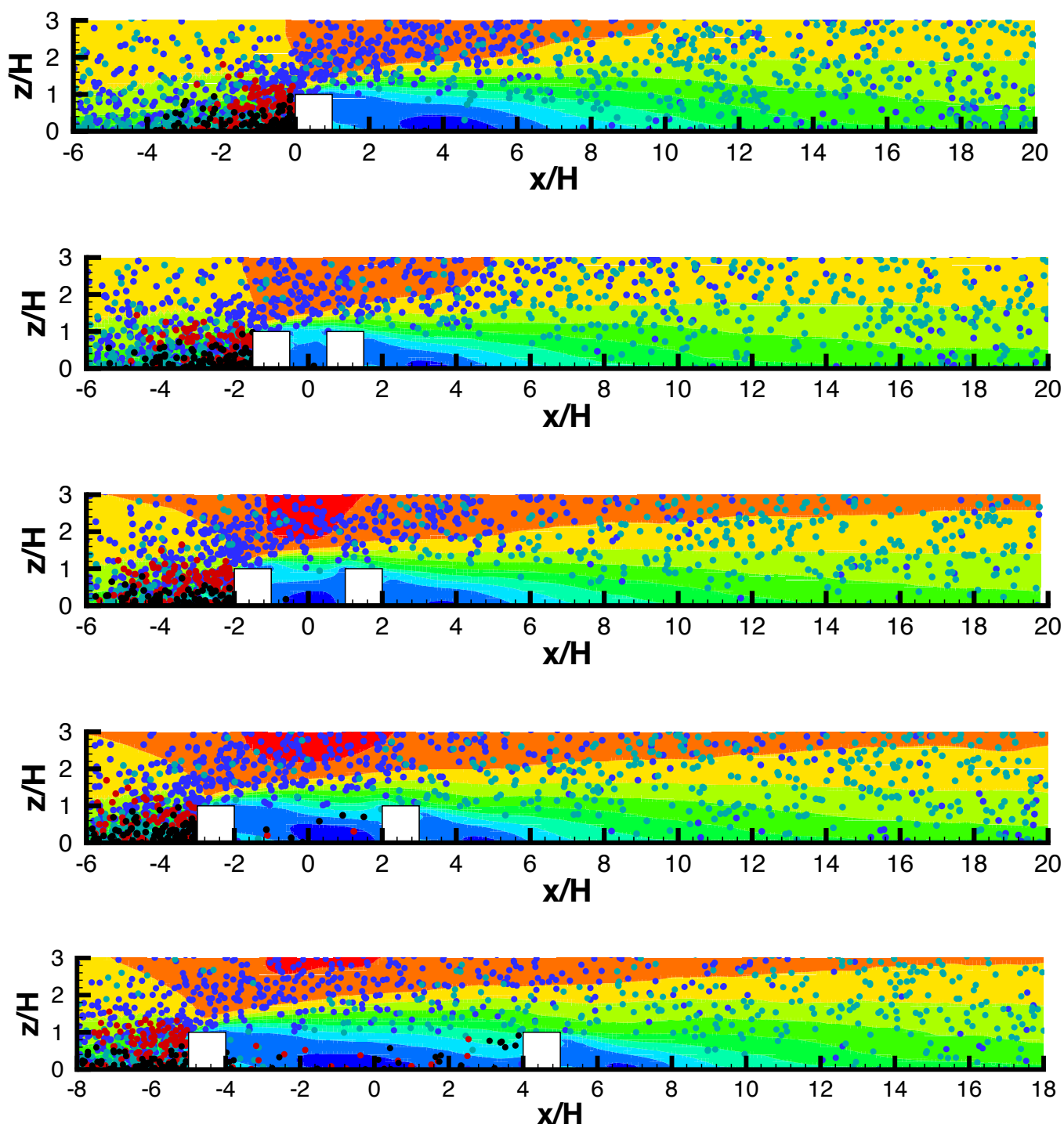

Figure 8. Particle position and fluid velocity isovalues. Color of the particles: Blue $-\left(u_{p}>0\right.$, $\left.w_{p}>0\right)$. Green - $\left(u_{p}>0, w_{p}<0\right)$. Red - $\left(u_{p}<0, w_{p}>0\right)$. Black - $\left(u_{p}<0, w_{p}<0\right)$. Colored average streamwise velocity isovalues are in $\mathrm{m} / \mathrm{s}$.

\subsection{Particle position}

Figure 8 illustrates the spatial distribution of a small percentage of particles $(5 \%$ of the followed particles at a given time) with the mean fluid flow streamwise velocity iso-contours in the background. Particles are coloured by their streamwise and vertical velocity components, $u_{p}$ and $w_{p}$ respectively. If $\left(u_{p}>0, w_{p}>0\right)$ particles are blue, for $\left(u_{p}>0, w_{p}<0\right)$ they are green, for $\left(u_{p}<0, w_{p}>0\right)$ particles are red and for $\left(u_{p}<0, w_{p}<0\right)$ they are black.

Particles accumulate before the first obstacle. Particles that are not trapped downstream, are deviated toward the main flow. Therefore, the first building acts as a trapping device upstream as well as a resuspension one since it projects particles to upper and faster moving flow regions. Blue regions on Figure 8 corresponding to low stream- 
wise velocity in the recirculation zone within the cavity are rather deprived of particles. Before the first obstacle, near the ground (below $\approx 0.5 H$ ) most particles are black inside the recirculation zone and green before. This corresponds to two groups of particles with $w_{p}<0$. Black particles $\left(u_{p}<0, w_{p}<0\right)$ have been trapped inside the recirculation zone. Green ones $\left(u_{p}>0, w_{p}<0\right)$ are near ground particles moving in the streamwise direction that meet the recirculation zone and get trapped. Above $z / H=$ 0.5 , most particles are red or blue corresponding to $w_{p}>0$. Since they have $u_{p}<0$ and $w_{p}>0$, red particles have probably rebounded on the obstacle, while blue particles $\left(u_{p}>0, w_{p}>0\right)$ are deviated by the obstacle and move streamwise and upward. After the first obstacle, most particles are first blue when they move upward and become green when they go back to the ground due to gravitational drift. After the first obstacle, all particles move streamwise, either upward because of the obstacle induced deviation (blue particles) or downward under the influence of gravity (green particles).

As the canyon opening increases, some particles fall within the cavity under the action of gravity and by the interaction of the mean flow and the recirculation zones. Moreover, by the random action of rebound and turbulence these particles remain trapped beneath the recirculation zone and might eventually be deposited. Here, some black particles might appear $\left(u_{p}<0, w_{p}<0\right)$, depicting the movement of sand trapped by the recirculation region that is downward and opposite to the streamwise direction. An estimation of the concentration increase in time could therefore be obtained inside the cavity.

For isolated and wake flows $(1 H, 2 H$ and $4 H)$ very few particles enter the cavity. For these cases, exchanges between the cavity (or the recirculation zone) and the upper layers are scarse, limiting the number of particles that enter the canyon. For the large separation $8 H$ case, the number of particles that fall within the cavity increases compared to the wake or isolated flow. For this large separation, an increase of solid particle exchange of the upper layer with the cavity is observed.

On Figure 9 the same particle distribution as the one given on Figure 8 is superposed with isocontours of Reynolds shear stress $u^{\prime} w^{\prime}$. As mentioned in section 6.3, a peak of positive $u^{\prime} w^{\prime}$ appears at roughly $1.2 H$ above the first obstacle. This region of high $u^{\prime} w^{\prime}$ values spreads and drifts away from the wall, illustrating the shear layer spread and vertical shift in the downstream direction. For $1 H$ and $2 H$ separations this shear layer stays above the canyon while it penetrates it for higher separations. Interestingly, particles just above the spreading shear layer are mostly blue $\left(u_{p}>0\right.$ and $\left.w_{p}>0\right)$. They move upwards and in the streamwise direction. Within the shear layer, particles are mostly green $\left(u_{p}>0\right.$ and $\left.w_{p}<0\right)$ meaning that they still move in the streamwise direction but are subjected to gravitational drift. Beneath the spreading high intensity $u^{\prime} w^{\prime}$ region, some black particles appear within the canyon for $4 H$ and $8 H$ separations, illustrating particles that are trapped within the canyon, beneath or within the recirculation region, as mentioned above. These particles present a downward and counter-streamwise movement $\left(u_{p}<0\right.$ and $\left.w_{p}<0\right)$. The shear layer illustrated by high values of $u^{\prime} w^{\prime}$ appears as a frontier between particles that fly above the canyon and particles that drift towards the canyon and get trapped within the recirculation.

\subsection{Mean concentration}

As stated in section 5 , at $x=6 H$ before the first obstacle, particles are injected by an exponential concentration profile classically obtained for saltation over flat sand beds (Creyssels et al., 2009; Durán et al., 2011).

Figure 10 shows particle mean concentration iso-levels. The concentration is obtained by counting the number of particles present within a box of size $H / 2 \times L_{y} \times H / 5$ at a given time. The mean concentration is then obtained by time averaging the concentration profiles for the five different obstacle configurations. Only the contribution 

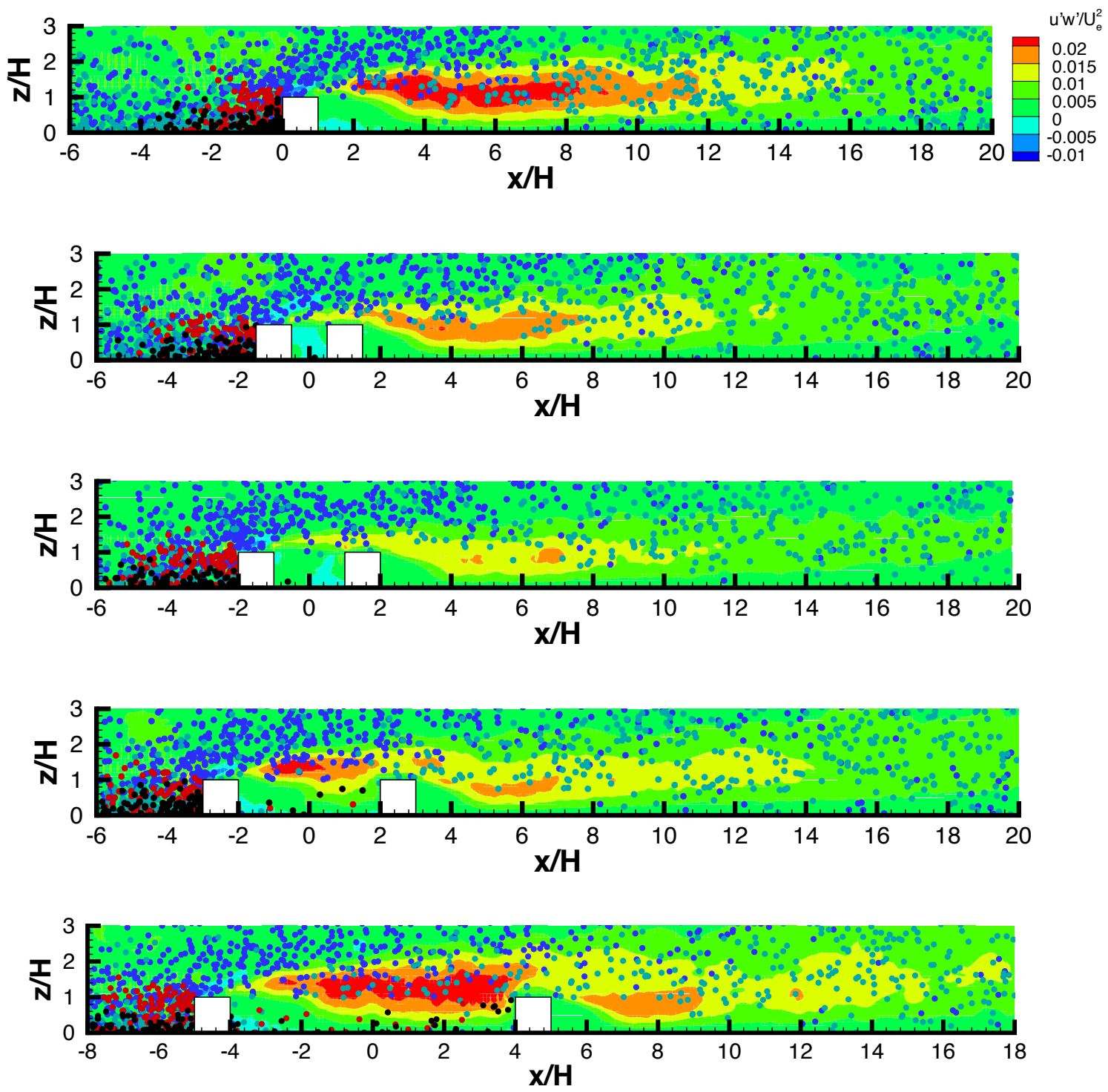

Figure 9. Particle position and fluid Reynolds stress $u / w /$ isovalues. Color of the particles: Blue - $\left(u_{p}>0, w_{p}>0\right)$. Green - $\left(u_{p}>0, w_{p}<0\right)$. Red - $\left(u_{p}<0, w_{p}>0\right)$. Black - $\left(u_{p}<0\right.$, $\left.w_{p}<0\right) . u^{\prime} w^{\prime}$ isovalues are normalized by $U_{e}^{2}$ as in Figure 4. 

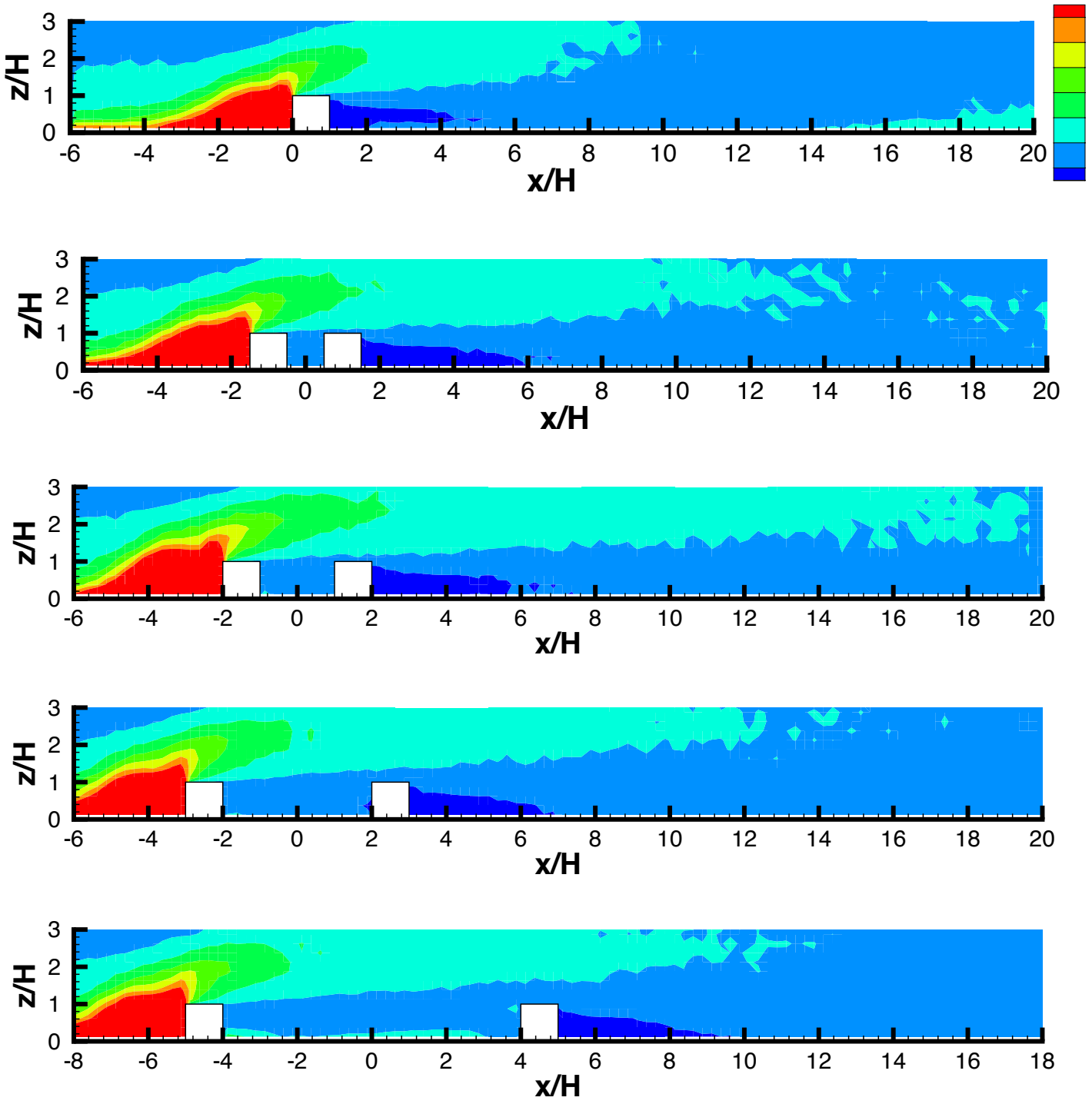

Figure 10. Mean particle concentration isovalues for different obstacle separations. From top to bottom - isolated case, $1 H, 2 H, 4 H$ and $8 H$ separations.

of particles transported by the flow is considered for computing the concentration. Namely, deposited particles are counted separately as described further in section 7.4. The particle concentration levels are plotted from $3 \mathrm{H}$ before the first obstacle until $20 \mathrm{H}$ and normalized by the maximum concentration $C_{m a x}$ in all the domain. For all cases, the presence of the first obstacle creates a barrier on which particles accumulate. This is observed on Figure 10 by the region of high particle concentration (red to yellow) just upwind of the first obstacle. Particles that are trapped in this region can be deposited by the action of the small recirculation zone present on the lee side of the obstacle. In addition to this, for all cases, the first obstacle deviates the particle trajectory inducing an upward moving and spreading concentration plume (green to light green).

For the isolated case, the concentration decreases after the obstacle but the particles above the obstacle continue their path. Due to dispersion, the particle plume becomes broader and then eventually due to gravity some particles deposit. Particles go 
back to the ground after the end of the large recirculation zone as it can be seen on Figure 10 starting from roughly $7 H$ after the obstacle. Thereafter the near wall concentration increases. This may show the return of an undisturbed saltation layer starting from $15 H$. Most particles that rebound on the wall or transit in the near-wall region after $7 H$ are submitted to classical flat sandy terrain saltation. From $7 H$ to $15 H$ particles have still enough energy to induce rolling until the re-establishing of the saltation layer. Nevertheless, only very low levels of solid particle concentration are observed within or beneath the recirculation zone which fails in capturing particles.

Between the two obstacles, the average concentration pattern depends on the distance between the obstacles. For the set of two obstacles with a $1 H$ and $2 H$ spacing, particles fly above the cavity and very few particles are trapped or deposited between the two obstacles. This could have been expected from the skimming flow regime where there is practically no interaction between the incoming flow and the cavity recirculation region.

In the $4 H$ and $8 H$ cases, the second obstacle is located after the streamwise position where particles begin to fall within the cavity (particles present a wall-normal position $z_{p}$ lower than $1 H$ ). This streamwise position where particles enter the cavity can be deduced from the isolated case on Figure 10. It is roughly $x \sim 2 H$. For the isolated case, as shown on Figure 8 via solid particle positions or on Figure 10 via green color, a plateau is observed between the end of the obstacle and the beginning of this drift zone. Therefore, the spacing between the two obstacles in the $4 H$ and the $8 H$ cases is large enough to allow particles to enter the cavity leading to increasing deposition. Small average concentration peaks are expected in this case near the ground between the two obstacles.

Average particle concentration profiles are presented for the different configurations in Figure 11. Profiles are normalized by the maximum concentration at each section. For all cases, profiles are presented at the particle injection section, at $1 H$ before the first obstacle within the small recirculation zone before this obstacle. For the isolated case, three profiles are plotted at $3 H, 5 H$ and $7 H$ after the obstacle. For the two obstacle cases, a profile is plotted at the middle between the two obstacles and at $2 H$ and $4 H$ after the second obstacle. For the $4 H$ and $8 H$ spacing cases, two additionnal profiles are plotted between the two obstacles.

For all cases, before the first obstacle, particles are trapped at the upstream wall corner producing a large concentration peak at the ground, whereas particles outside this region fly above the obstacle. This is observed by a spreading peak of average particle concentration for $z / H>1$. The large concentration peak at the ground corresponds to particles that are still transported by the flow or rebounding on the surface. As stated above and described further in section 7.4, deposited particles are accounted for separately.

For the isolated obstacle, at $x / H=3$, the concentration is almost equal to zero under the height of the obstacle and the average concentration peak is located approximately around $2 H$ above the wall.

From $3 H$ to $7 H$, due to dispersion, the concentration profiles widen and the height of the peak increases. On the third profile, at $7 H$, particles begin to deposit and a very low level secondary concentration peak appears near the ground. This secondary peak is much lower than the spreading particle plume above the canopy, implying that in this case the obstacle plays the role of dispersion rather than it enhances deposition.

For the two obstacle cases in the skimming regime, namely $1 H$ and $2 H$ separations, the same very low level secondary concentration peak is observed at the ground within the cavity. Nevertheless, both for the isolated and the skimming case the ground level 

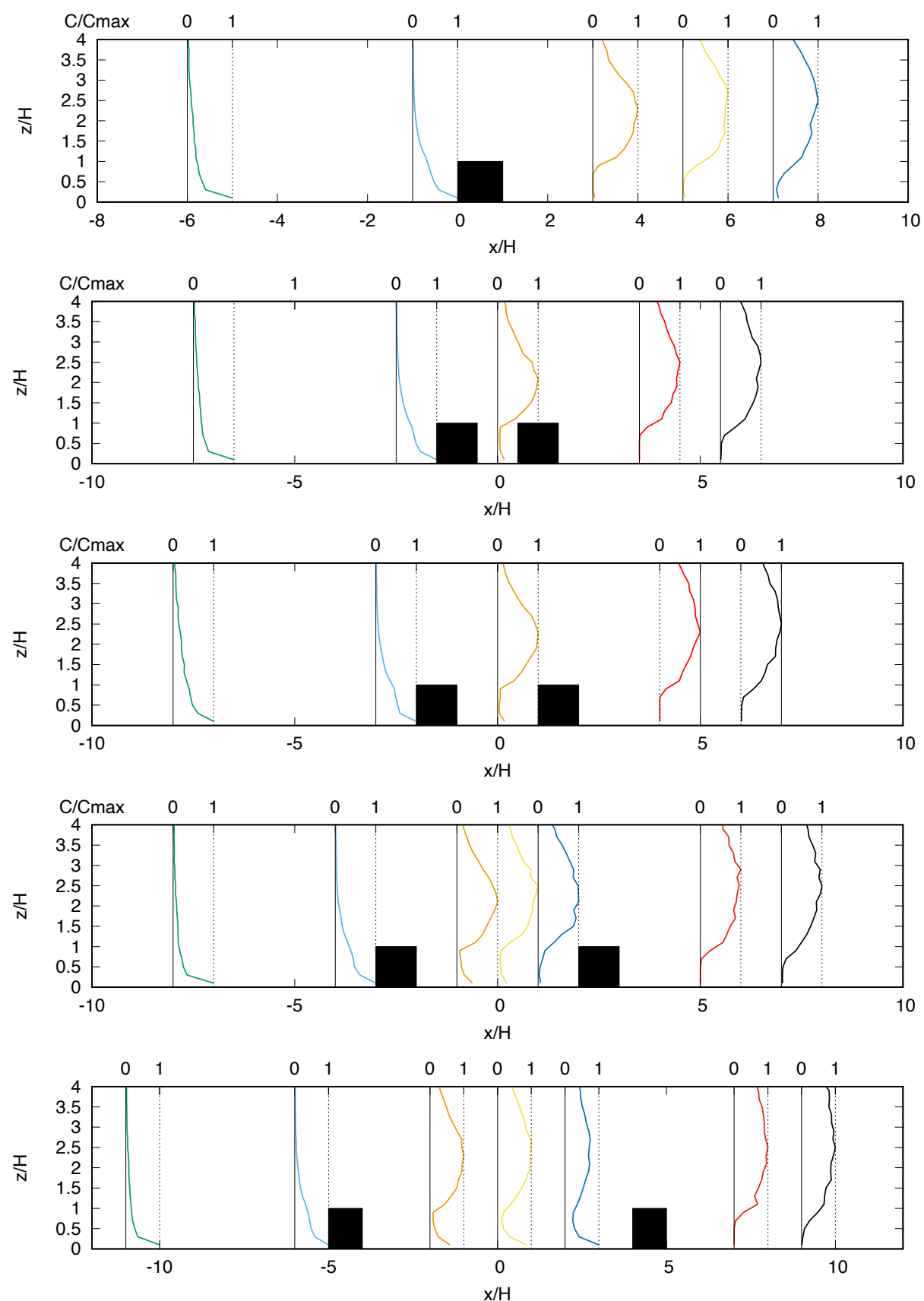

Figure 11. Mean concentration profiles for an isolated obstacle and for obstacles with different spacings $1 H, 2 H, 4 H$ and $8 H$ from top to bottom. 
concentration of particles within the cavity is lower than for the two other cases namely $4 H$ and $8 H$.

For the skimming flow, after the second obstacle, profiles are similar to the isolated case with an even higher value of the concentration peak above the cavity and even lower levels of particles in the near wall region. This suggests that as for the skimming flow, the cavity is seen as a single large obstacle by the incoming particle saltation layer. This layer is partly trapped upstream and partly deviated towards the main flow above the cavity.

For $4 H$ and $8 H$ spacings, a small but non negligible concentration peak appears on the wall inside the cavity. This corresponds to particles trapped within the primary recirculation region that deposit on the wall under the influence of gravity. For the $8 H$ separation, the peak on the wall in the middle of the cavity is almost as large as the concentration peak above the obstacles. Particles are trapped in the recirculation zone between the two obstacles and fall to the wall under the influence of gravity. The internal friction velocity is not high enough to reinitiate suspension. The wake flow and transitional isolated regimes allow particle deposition by upstream flow penetration within the cavity. This emphasizes the ability of the wake and transitional isolated flows to trap particles beneath the primary recirculation.

As for the skimming flow, after the downstream obstacle in the wake and transitional isolated flows, particle concentration is practically zero near the ground and presents a spreading peak at roughly $2 H$ from the wall. This suggests deviation of the remaining saltation layer by the presence of obstacles.

Figure 12 shows the streamwise evolution of the maximum concentration and its vertical position. The maximum concentration is adimensionalized by its maximum in the domain. Highest particle concentrations are obtained before the first obstacle by particle accumulation upstream a vertical barrier. For all cases, further downstream, above the cavity or beyond, the maximum concentration used for the normalization of the previously analysed Figure 11 decreases. The maximum concentration decreases as well with the canyon opening. This shows that the spreading of the saltation layer that has been deviated by the first obstacle grows as the opening between the obstacles is increased.

Furthermore, Figure 12 illustrates that the vertical position of the maximum concentration is close to the wall before the first obstacle. This is due to the initial concentration profile and the blockage produced by the first upstream obstacle. Downstream, the height of the maximum concentration switches above the cavity emphasizing the saltation layer deviation discussed above. The height of $C_{\max }$ slightly increases with the spreading of the particle plume. For the largest separation $(8 H)$ a switch toward the wall in the vertical position of the maximum concentration is observed in the cavity for $0<x / H<$ 3 , roughly. Namely, for the $8 H$ spacing, a second high peak appears near the wall. Its intensity remains smaller than the peak above the obstacle until approximately the middle of the cavity. Further downstream, this wall concentration peak exceeds the high concentration levels observed above the cavity as particles settle toward the wall. As the second obstacle is approached for the $8 H$ case separation, the maximum concentrations above the cavity increase compared to the near wall concentration accounting for the transport of particles by the main flow that circumvent the obstacles. The position of the $C_{\max }$ peak switches back to $z / H \sim 2$. For all two obstacles cases, after the cavity $C_{\max }$ is around $z / H \sim 2$. After the second obstacle, this position first increases as a consequence of the saltation layer deviation. It then starts to slightly decrease from $x / H \sim 15$ and further downstream because of particle gravitational settling. It is only for the isolated case, starting from $x / H \sim 14$ that the maximum concentration peak falls back to the wall as in the incoming initial concentration profile. To gain insight on solid particle transfers between the main flow and the cavity, the particle streamwise and vertical velocity profiles are presented in the next section. 

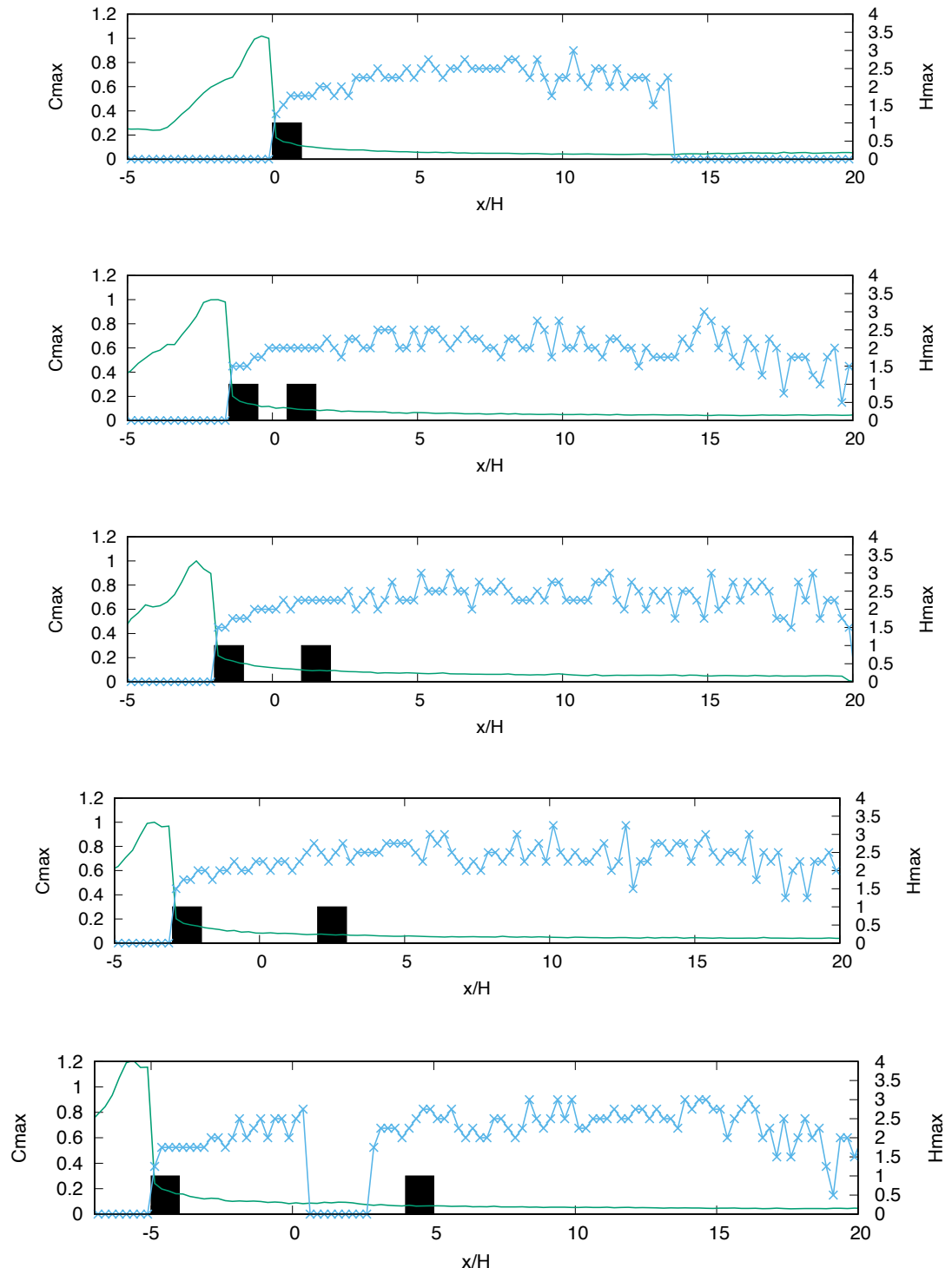

Figure 12. Longitudinal profiles of the maximum concentration level (green line, left scale) and of the height of this maximum (blue line, right scale) for an isolated obstacle and for obstacles with different spacings $1 H, 2 H, 4 H$ and $8 H$ from top to bottom. 

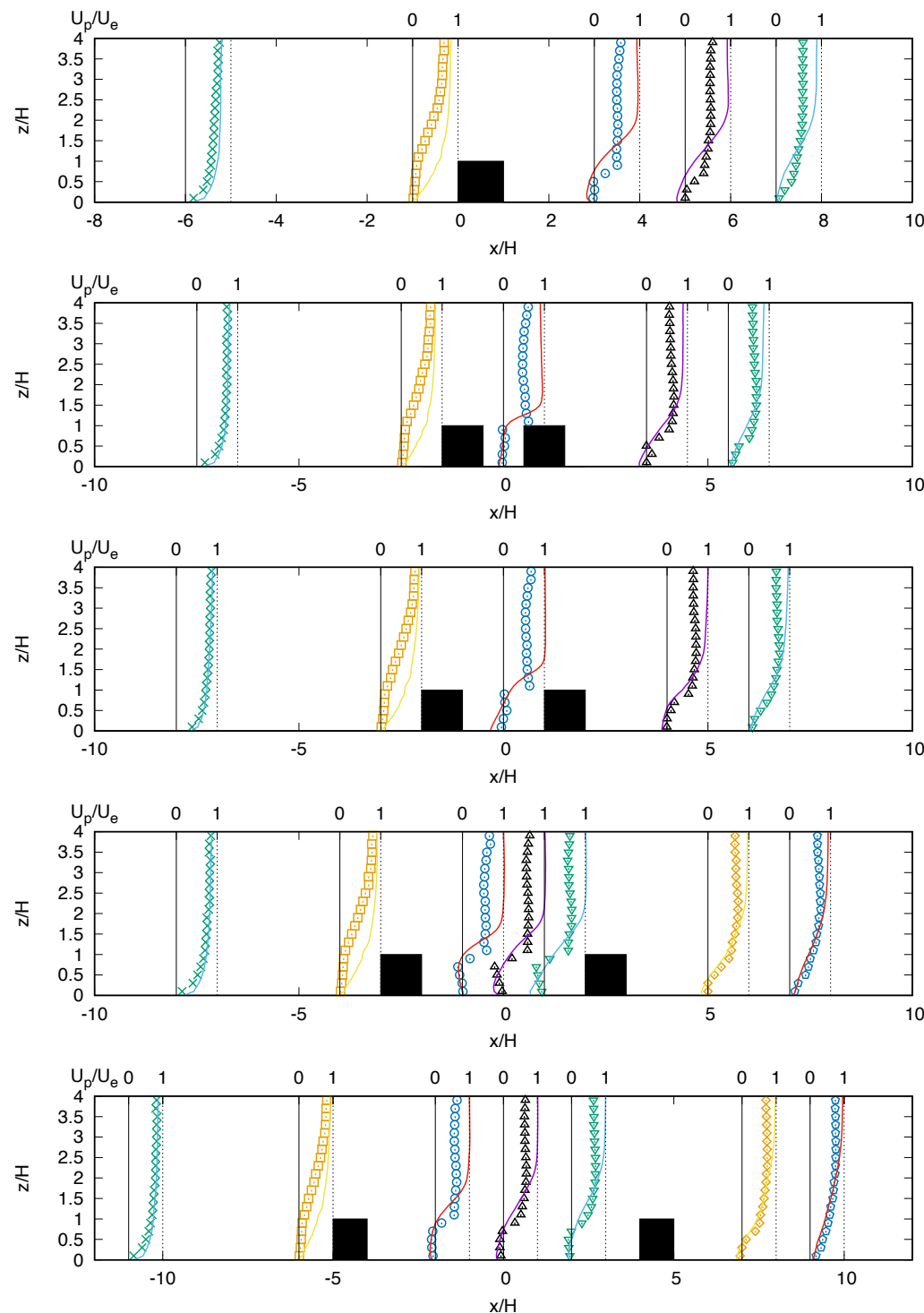

Figure 13. Mean streamwise velocity profiles for an isolated obstacle and for obstacles with different spacings $1 H, 2 H, 4 H$ and $8 H$ from top to bottom. Lines - fluid. Symbols - particles.

\subsection{Particle velocity}

The streamwise and vertical particle velocity profiles are shown in the Figures 13 and 14 for the five different obstacle configurations. Wind velocity profiles at the same location are added for comparison. For all plots, the symbols represent the solid particle velocity, whereas the lines represent the fluid velocity. Plots are presented at the same locations as the concentration profiles shown on Figure 11. The mean particle velocity profiles are obtained by averaging the particle velocity on all particles present within a box of size $H / 2 \times L_{y} \times H / 5$ at a given time. This gives an eulerian reconstruction of the solid particle velocity profiles which are then averaged additionally in time.

The first profiles are plotted at the inlet and at $1 H$ before the first obstacle. At the inlet, the velocity is set to the fluid velocity. The profile at $1 H$ is located in a zone of high particle concentration. At this location, the profiles are similar for the five con- 

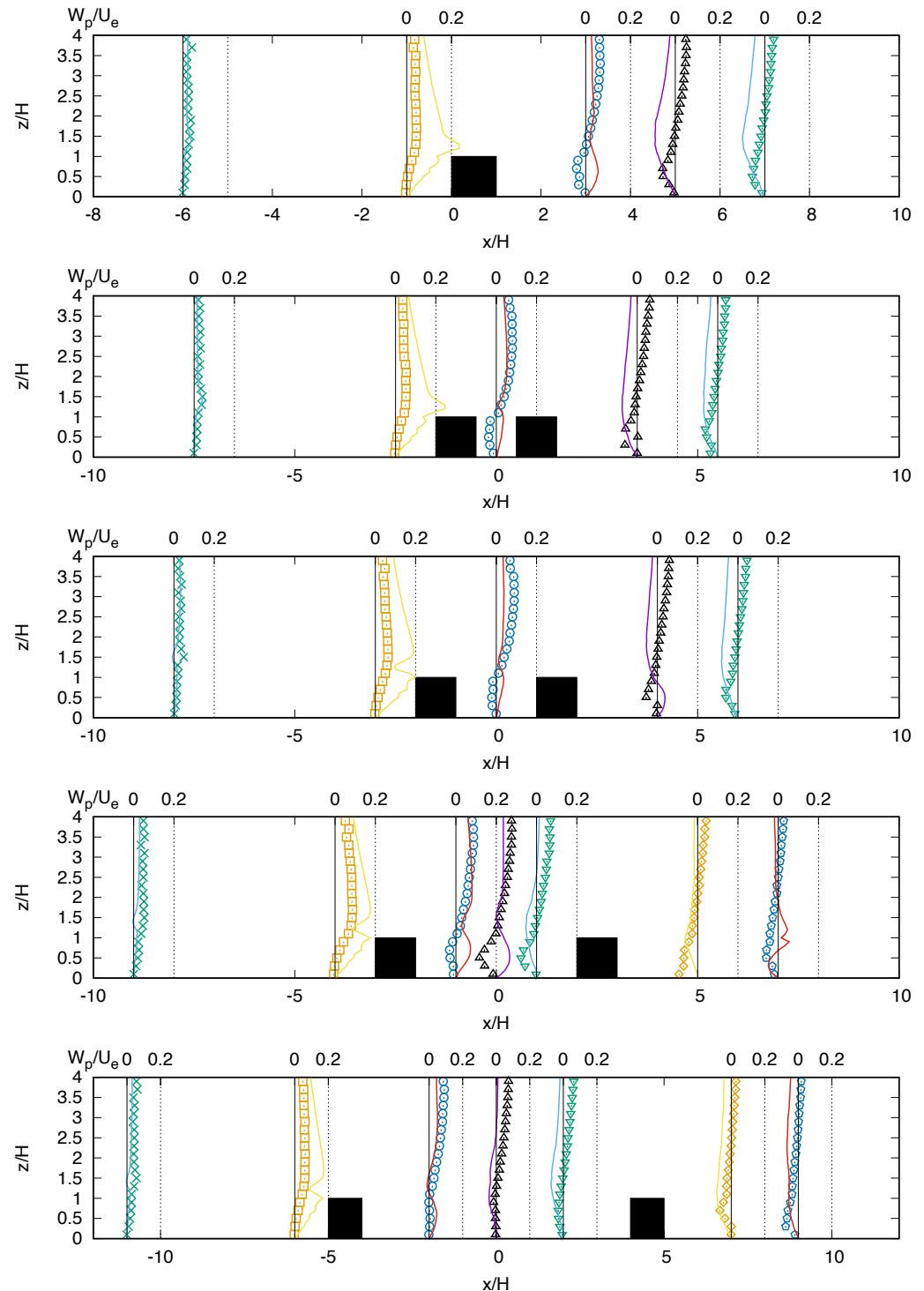

Figure 14. Mean vertical velocity profiles for an isolated obstacle and for obstacles with different spacings $1 H, 2 H, 4 H$ and $8 H$ from top to bottom. Lines - fluid. Symbols - particles. 
figurations. A great amount of particles is trapped just before the first obstacle where the streamwise solid particle velocity is zero on average for $z / H<1$. Before the first obstacle, the solid particle streamwise velocity is always lower than the fluid one. Solid particles lag on the average streamwise fluid velocity because of their inertia. Before the first obstacle, the vertical fluid velocity is small near the ground. It then increases to reach a peak just above the obstacle at $z / H=1.2$ as the fluid flows upwards to circumvent the obstacle. The vertical velocity of the solid phase follows the same pattern but due to particle inertia, the particle velocity remains smaller than the vertical fluid one. Above the obstacle, the streamwise particle velocity increases and reaches the wind velocity at $z / H=3$, while the vertical velocity decreases.

For the isolated obstacle, the first section after the obstacle (at $3 H$ ) is located within the big recirculation zone. The concentration profiles show that very few particles are present is this zone (Figure 11). The streamwise fluid velocity is negative and the particle velocity is almost zero near the wall. The vertical fluid velocity is positive since the recirculation zone spreads above the roof top, whereas the particle velocity is negative. Particles within the recirculation zone have been brought by the reversal flow. Here, the streamwise velocity is not strong enough and particles fall to the wall under the influence of gravity. At $x / H=5$ and 7 , the vertical velocity is negative for both the fluid and the particles for $z / H<1$. The flow is directed slightly towards the wall up to the reattachment point. Particles follow this pattern and will be deposited. Above $z / H=$ 1 , for $x / H=5$ and 7 , the average fluid vertical velocities are still negative, whereas mean particle vertical velocity profiles are positive. This points out the long lasting effect of the upward projection of the saltation layer caused by the isolated obstacle.

The same conclusions can be drawn for all two obstacle configurations. The streamwise particle velocity is almost zero between the obstacles in the primary recirculation zones. Above the cavity, streamwise particle velocity profiles are always lower than the corresponding fluid velocity profiles because of the particle inertial lag. Within the cavity, the particle vertical velocity is negative indicating solid particle sedimentation in low streamwise fluid velocity regions. Above the cavity, particle vertical velocity profiles are positive and higher than the fluid vertical velocities. The deviated saltation layer is projected upwards and still moves under the influence of this deviation. Further downstream, particles may start to recover and to adjust to the average vertical fluid velocity. This is seen by the reduced difference between the solid and the fluid average velocity profiles at the furthest downstream position.

After the second obstacle, the streamwise particle velocity is close to zero near the wall while the vertical component is negative, illustrating particle deposition in low streamwise fluid velocity regions. Above $z / H \sim 1$, particles are slower than the average fluid in the streamwise direction but move faster than the fluid in the upward vertical direction. Once again, this is a consequence of the vertical deviation of the saltation layer that has to circumvent the obstacles.

\subsection{Particle deposition and emission}

Particle deposition and entrainment rates are presented in Figure 15 for the five different configurations.

The deposition (emission) rate is the number of particles that deposit (take-off) per square meter and per unit time. As described in section 4, a particle that impacts the wall may rebound or remain on the wall according to the probability given by equation 6. Particles that remain on the wall are counted for the deposition rate. All the profiles are normalized by the maximum deposition $D_{\max }$. Normalized deposition $D / D_{\max }$ is given on the lefthand side while normalized emission $E / D_{\max }$ is on the righthand side of Figure 15. Emission is at least 10 times smaller than deposition as seen by the range of the left and righthand side axis on Figure 15. In this study, there are no available par- 

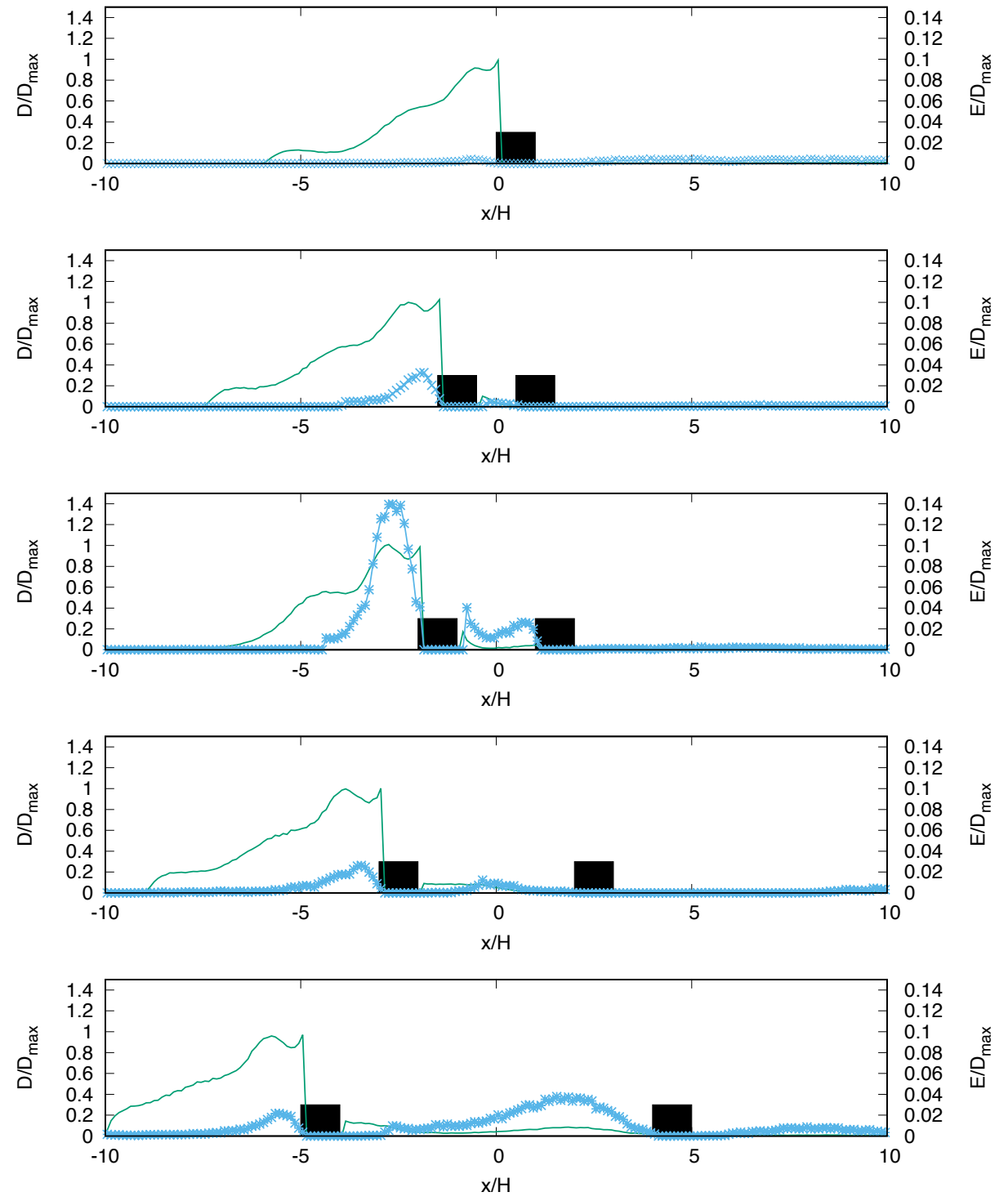

Figure 15. Particles deposition and emission zones for an isolated obstacle and for obstacles with different spacings $1 H, 2 H, 4 H$ and $8 H$ from top to bottom. Lines - deposition. Symbols emission. 
ticles on the ground. Take-off may take place only in regions where deposition has previously been encountered.

Globally, high particle deposition zones coincide with high concentration zones on the wall. For all configurations, there is a rather high deposition peak just before the first obstacle. Due to the blocking effect of the first obstacle, most particles with $z_{p}<H$ are trapped within the small recirculation bubble and deposited. For skimming flow (obstacle separation of $1 H$ and $2 H$ ), there is also a peak of emission before the first obstacle. In this region many particles have been deposited, and a small part of them is reentrained by the flow.

After the first obstacle, particle deposition decreases abruptly. In the primary recirculation zone, deposition mostly takes place on the lee side of the upwind obstacle. Deposition is locally higher for skimming flows $(1 H$ and $2 H)$ but it spreads further downstream in the case of wake and isolated flow $(4 H$ and $8 H)$. Few particles take-off in the primary recirculation in the skimming flow case $(2 H)$ and in the transitional isolated flow case $(8 H)$. These emission rates remain small compared to deposition.

For the $8 H$ case, the tertiary recirculation zone on the windward side of the second obstacle plays a favorable role for deposition as a second small bump on the streamwise evolution of deposition is observed around $x / H \sim 2$. However, in this low streamwise fluid velocity region, emission is half the deposition, illustrating that half of the deposited particles still take-off.

Figure 16 (right) illustrates the evolution of the maximum deposition $D_{\max }$ used for normalizing the deposition in Figure 15 with the street canyon opening. Values of the maximum deposition $D_{\max }$ shown in Figure 16 (right) are normalized by the maximum deposition obtained for the isolated case $D_{\text {max }, \text { isolated }}$ since the deposition computed here represents only a number of particles per square meter and unit time. Although a very similar behavior of the normalized $D / D_{\max }$ deposition between all cases has been observed from Figure 15, the highest maximum deposition is obtained for the isolated case. $D_{\max } / D_{\max , \text { isolated }}$ drops as the separation is increased in the skimming flow regime. For wake flow ( $4 H$ separation) and transitional isolated flow ( $8 H$ separation) $D_{\max } / D_{\max , \text { isolated }}$ increases with higher openings. However, $D_{\max } / D_{\max \text {, isolated }}$ remains smaller than 1 implying that $D_{\max }$ still presents lower values than in the isolated case. The lowest maximum deposition is obtained here for the $2 \mathrm{H}$ skimming flow street canyon opening.

Figure 16 (left) presents the local time and space average deposition $D$ between $x_{1}$ and $x_{2}$ (Table 3 ) and within the canyon, for each configuration, normalized by the deposition of the isolated case $D_{\text {isolated }}$. When averaging is performed between $x_{1}$ and $x_{2}$, the lowest deposition is obtained for skimming flow $(2 H)$, as for $D_{\max }$. For $4 H$ separation, deposition is higher than for the isolated case when the average value of the deposition is considered between $x_{1}$ and $x_{2}$. This clearly corresponds to the expected solid particle behavior described in section 6.4 by analyzing the STA friction velocity $u_{*}^{m} / u_{*}^{0}$ averaged between $x_{1}$ and $x_{2}$ (Figure 7 ). Namely, Figure 7 showed that when averaged between $x_{1}$ and $x_{2}$, the highest STA friction velocity $u_{*}^{m} / u_{*}^{0}$ was obtained for skimming flow $(2 H)$. Therefore, the lowest deposition is expected in this case. If we now consider only the average within the canyon, deposition drops as the spacing is increased for skimming flow. For the wake and transitional isolated flows, the average deposition within the canyon increases with obstacle separation. Once again, this is in accordance with the evolution of $u_{*}^{m b} / u_{*}^{0}$ described in Figure 7 . When the friction velocity is averaged only within the canyon $\left(u_{*}^{m b} / u_{*}^{0}\right)$, the overall friction is reduced. The highest reduction is obtained for the skimming flow since the incoming flow circumvents the obstacles without penetrating the canyon. As the separation is increased, in the wake flow and the transitional isolated cases, the friction reduction is less pronounced. In terms of total particle deposition within the canyon, this behaviour of $u_{*}^{m b} / u_{*}^{0}$ implies a minimum depo- 

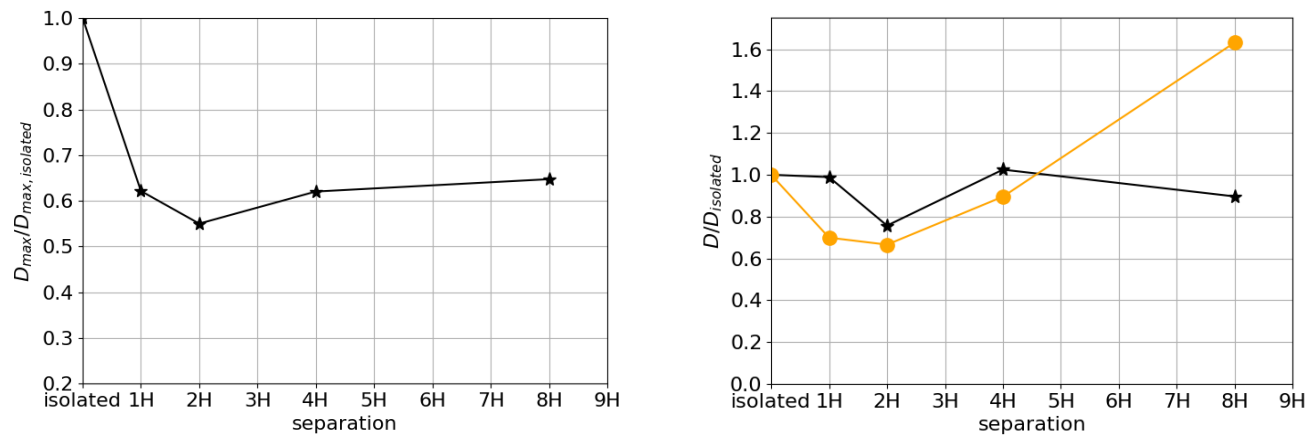

Figure 16. Left - Evolution of $D_{\max }$ with obstacle separation. Right - Local time and space average deposition rate normalized by the deposition rate of the isolated case as function of the street canyon opening. Stars - Average between $x_{1}$ and $x_{2}$ (Table 3). Circles - Average only within the canyon.

sition for skimming flow and a steady increase of deposition thereafter (for wake and transitional isolated flows). The $8 H$ separation case leads to higher deposition within the canyon than the isolated case.

\subsection{Vertical and horizontal saltation mass flux}

The streamwise saltation mass flux $q_{x}(x, z)\left(\mathrm{kg} \cdot \mathrm{m}^{-2} \cdot \mathrm{s}^{-1}\right)$ is computed as follows:

$$
q_{x}(x, z)=\frac{1}{V} \sum_{V} m_{p} u_{p}
$$

where $m_{p}$ and $u_{p}$ are the mass and the streamwise velocity of the particles present in the volume $V$. The vertical profiles of the mass flux are presented in Figures 17 for the five configurations. The mass fluxes have been multiplied by 1000 . Plots are presented at the same locations as the concentration profiles on Figure 11.

In accordance with our previous observations, for all the cases studied here, the most notable trend of the streamwise saltation mass flux is the presence of a peak above the top of the obstacles at roughly $2 H$. Obstacles deviate an incoming saltation layer by imposing the circumvention of the obstacle by particles and by the flow. The streamwise saltation mass flux peak spreads and decreases in intensity for all the studied configurations in the streamwise direction. As the cavity opening is increased, the spread of the peak is amplified and the value of the maximum is further decreased. At similar $x / H$ streamwise positions, lower saltation mass flux values are obtained when canyon openings are higher. Therefore, the lowest level of the streamwise saltation mass flux is obtained downstream of the $8 H$ separation case corresponding to the limit between isolated and wake flow. Within the canyon, near the wall $q_{x}(x, z)$ is mostly zero. Few particles are found within the canyon. Moreover, close to the wall their streamwise velocity is zero canceling the streamwise flux. Once again, as stated above, obstacles also shelter wall particles underneath the primary recirculation zone. This is confirmed by the distinct streamwise saltation flux reduction within the canyon compared to the incoming profiles.

To compare the impact of the different configurations studied here on the long range streamwise saltation flux, Figure 18 (Left) gives the streamwise evolution of the horizontal flux $q_{x}(x, z)$ at $z / H=0.5$ after the first obstacle. All curves begin at the first obstacles, therefore between $x / H=0$ and 1 , the flux is equal to zero since there are 

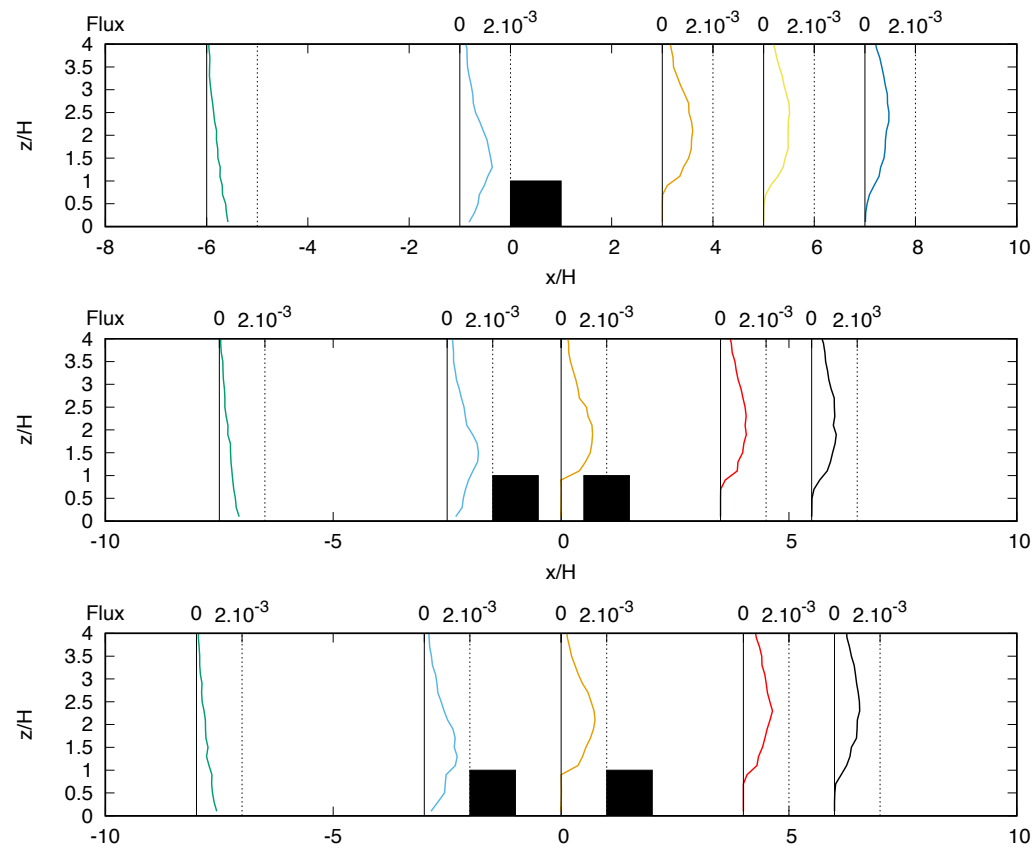

$\mathrm{x} / \mathrm{H}$
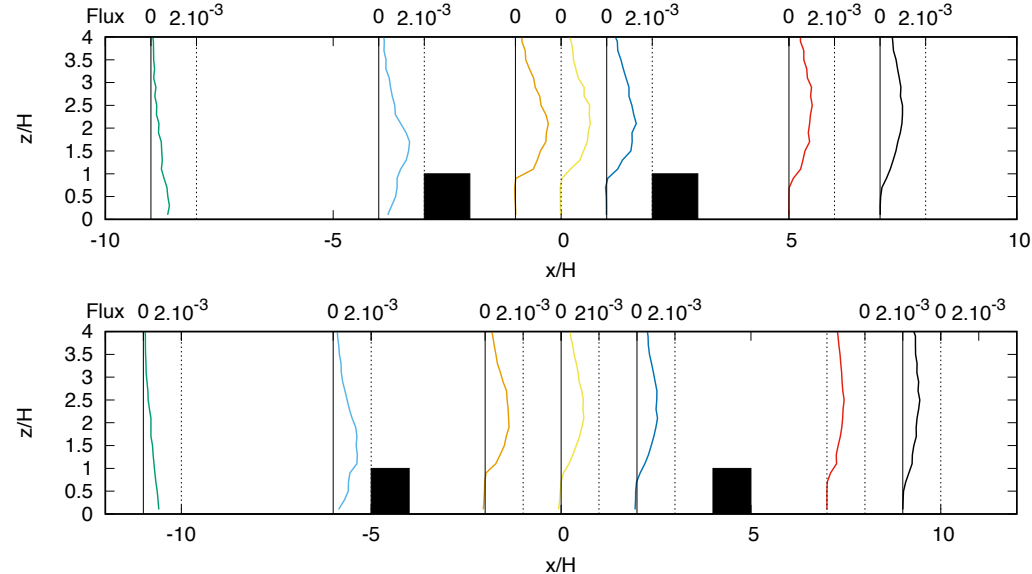

Figure 17. Saltation mass flux $q_{x}(x, z)$ for an isolated obstacle and for obstacles with different spacings $1 H, 2 H, 4 H$ and $8 H$ from top to bottom. The mass flux has been multiplied by 1000 . 

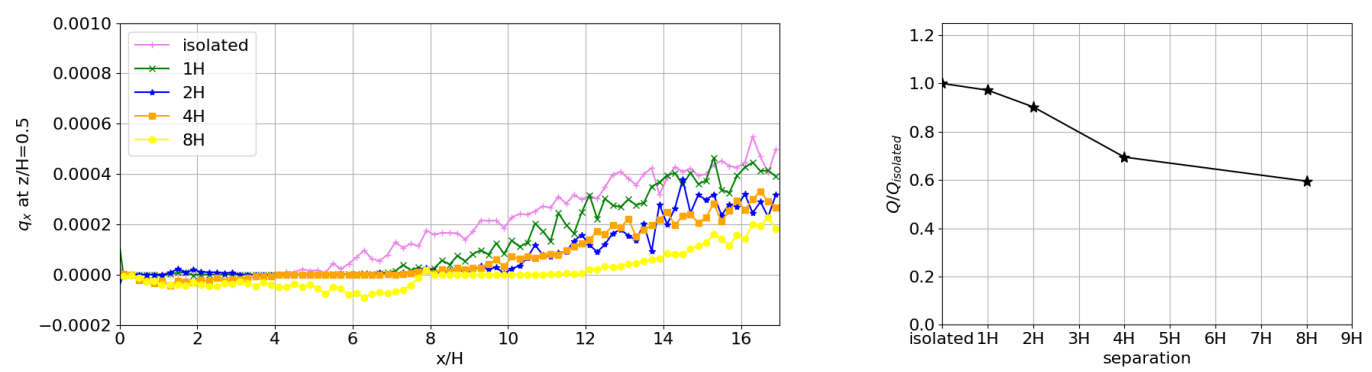

Figure 18. Left - Longitudinal evolution of the saltation flux $q_{x}(x, z)$ at $z / H=0.5$. Right Time and space averaged streamwise saltation flux at $z / H=0.5$ normalized by the isolated case average flux.

no particles inside the obstacle. The flux is also zero at the streamwise position of the second obstacle.

For the isolated case, at this height, the flux is very small after the obstacle and becomes negative in the recirculation zone. It then increases after the recirculation zone. For the two obstacle cases, the location where the flux begins to grow shifts downstream with the distance between the obstacles. Far from the obstacles for $x / H>8$, the highest flux is obtained for the $1 H$ separation case. As the spacing between the obstacles increases, the flux is reduced. The highest flux is achieved for the two skimming flow regimes. The smallest flux is obtained for the two obstacle case with $8 H$ separation, namely the case at the limit between the wake flow and the isolated flow regime, or transitional isolated flow.

Figure 18 (Right) shows the streamwise average of the longitudinal saltation mass flux $q_{x}(x, z)$ at $z / H=0.5$ given on the lefthand side of the same figure. This streamwise average of $q_{x}(x, z)$ at $z / H=0.5$ is denoted here by $Q$. The space and time average flux at $z / H=0.5, Q$, has been normalized by the isolated case value for comparison $\left(Q_{\text {isolated }}\right)$. As expected from the left-hand side evolution of the saltation mass flux shown on Figure 18, the space average of the saltation flux at $z / H=0.5(Q)$ is always smaller than for the isolated case $\left(Q / Q_{\text {isolated }}<1\right)$ and it decreases with street canyon opening. For the two obstacle cases, the highest space and time average saltation flux $Q$ is obtained for skimming flow and the lowest one is achieved for high separations corresponding to the transitional isolated case. These observations from Figure 18 confirm previous conclusions based on the average friction velocity between $x_{1}$ and $x_{2}, u_{*}^{m} / u_{*}^{0}$ (Table 3 and Figure 7) and deposition (Figure 16) stating that the highest STA friction velocity $u_{*}^{m} / u_{*}^{0}$ is obtained for skimming flows leading to the lowest deposition and therefore the highest streamwise saltation flux at $z / H=0.5$.

The results presented here give a first indication on the tests that can be conducted when saltation flux reduction is aimed at. They also illustrate a configuration that can be used for reducing streamwise saltation fluxes and therefore developing strategies for desertification control. One might think that with more recirculation zones higher saltation flux reductions are obtained. The more recirculation zones, the easier it would be to shelter wall particles from the incoming flow. However, obstacles should be disposed in such a way to avoid saltation layer deviation and projection to high streamwise velocity regions of the flow. 


\section{Conclusion}

In this paper, particle transport within a turbulent boundary layer in the presence of one or several squared cross-section obstacles is studied to evaluate the impact of construction spacings on sand particle fluxes. One or two square obstacles with different separations are disposed perpendicularly along a turbulent boundary layer. A constant flux of sand particles with an exponential concentration vertical profile, is injected at the inflow. The influence of the obstacles and their separation on particle transport, deposition and take-off is investigated.

The fluid flow is resolved using a large-eddy simulation. Solid particles are tracked in a Lagrangian way. The particle lift is related to events of strong sweep turbulent structures evaluated locally and instantaneously by the LES. Special focus is put on the prediction of the recirculation zones. By observing the simulated flow patterns, the different configurations studied here are sorted according to the classification of Oke (1988), namely isolated flow (one obstacle), skimming flow ( $H$ and $2 H$ separation), wake flow $(4 H)$ and transitional wake to isolated flow $(8 H)$. Mean and rms fluid velocity obtained by LES are in good agreement with the experimental profiles of Simoens et al. (2007) although the Reynolds number is smaller in the simulations. Fluid velocity profiles confirm the existence of the primary recirculation region within the canyon and behind the isolated obstacle and point out the presence of a spreading shear layer at roughly the roof top. The shear layer spreads and weakens as the canyon opening is increased. The fluid velocity rms profiles illustrate that for the wake $(4 H$ spacing $)$ and transitional isolated (8H spacing) flow regimes, the shear layer slightly penetrates the region within the obstacles $(z / H<1)$. The streamwise evolution of the local time averaged friction velocity is used to discuss potential particle trapping regions within the primary recirculation zone. Within the canyon, the global space and time average of the friction velocity decreases compared to the inlet boundary layer friction velocity value suggesting that obstacles reduce friction and provide shelter for particle deposition.

Particle distribution and concentration profiles show that particles accumulate before the first obstacle. Some particles are also deviated toward the main flow. Thus, the first obstacle acts as a trapping device as well as a resuspension one since it projects particles to upper and faster moving flow regions. For canyon openings of $4 H$ and more, some particles penetrate the gap between the obstacles and a secondary concentration peak appears on the wall in this region. However, for all configurations the highest concentration is observed within the shear layer just above the roof top of the first obstacle. This region of high concentration spreads downstream and with canyon opening.

Particle streamwise velocity profiles are always lower than the corresponding fluid velocity profiles because of the particle inertial lag. Within the cavity, the negative particle vertical velocities indicate solid particle sedimentation in low streamwise fluid velocity regions. Above the cavity, positive particle vertical velocities are higher than the negative fluid vertical velocity. This illustrates how the deviated saltation layer is projected upwards and moves under the influence of the deviation downstream.

Particle deposition is particularly high in the upstream region just before the first obstacle where most particles get trapped. In the primary recirculation zone, the deposition rate is locally higher in the skimming flow regime $(1 H$ and $2 H)$ but it spreads further downstream in the case of wake and transitional isolated flow $(4 H$ and $8 H)$. Few particles take-off in the primary recirculation in the skimming flow case $(2 H)$ and in the transitional isolated flow case $(8 H)$. However, these emission rates remain small since here, only deposited particles can take-off.

In order to compare the impact of the different configurations on particle transport, the streamwise saltation mass flux $q_{x}$ is computed. It presents a spreading peak above the top of the obstacles. This spreading increases with obstacle separation. The 
lowest level of the streamwise saltation is obtained for the limiting case of transitional isolated and wake flow ( $8 H$ separation). Obstacles deviate the incoming saltation layer. However, they also shelter particles that may be on the wall underneath the primary recirculation region.

Observations based on the average friction velocity between $x_{1}$ and $x_{2}$ (Table 3 and Figure 7) and the deposition rate (Figure 16) point out that the highest space and time average friction velocity is obtained for the skimming flow case leading to the lowest deposition rate and therefore the highest average streamwise saltation mass flux at $z / H=$ 0.5. In the skimming flow regime, the incoming fluid does not penetrate into the cavity and therefore sees the two consecutive obstacles as one big obstacle. Higher friction is thus obtained in this case leading to lower deposition and higher streamwise saltation flux compared to the higher canyon openings (wake flow and transitional isolated flow regimes). In previously studied successive Gaussian hill configurations (G. Huang et al., 2018,2019 ), it has also been observed that wake flow allows more accumulation than in the skimming flow regime. The rather similar qualitative behavior of these two very different aerodynamic configurations (successive Gaussian hills versus street canyons) points out the interest of the general classification proposed by Oke (1988). The average friction velocity, deposition rate and streamwise saltation flux computed in this study for the three flow regimes, as sorted by Oke (1988), provide a set of boundary conditions useful in other meso-scale simulations.

The results presented here give a first indication on the impact of obstacles and obstacle separation on particle transport by saltation. Average friction velocity values within the canyon are reduced by the presence of obstacles. Obstacles can also trap particles or create low streamwise velocity regions where particles are sheltered and might deposit. However, if obstacle separation is not accurately devised, the first incoming obstacle might simply project the saltation layer to higher streamwise velocity regions and generate longer range saltation. Data presented here can be used for calibration of large scale sand particle transport models. Nevertheless, a larger parametric study and the simulation of more realistic obstacles used to control desertification will be performed in future work.

\section{Appendix A Grid convergence}

Tests with two higher and one coarser grid resolution have been performed. Details of the tests are given in Table A1.

Table A1. Parameters for grid convergence tests

\begin{tabular}{lccc}
\hline Case & $\frac{\Delta x}{H}$ & $\frac{\Delta y}{H}$ & $\frac{\Delta z_{\text {min }}}{H}$ \\
\hline Reference & 0.1 & 0.2 & 0.1 \\
\hline Coarse grid & 0.2 & 0.4 & 0.1 \\
\hline Fine grid 1 & 0.05 & 0.1 & 0.1 \\
\hline Fine grid 2 & 0.1 & 0.2 & 0.005 \\
\hline Fine grid 3 & 0.1 & 0.1 & 0.005
\end{tabular}



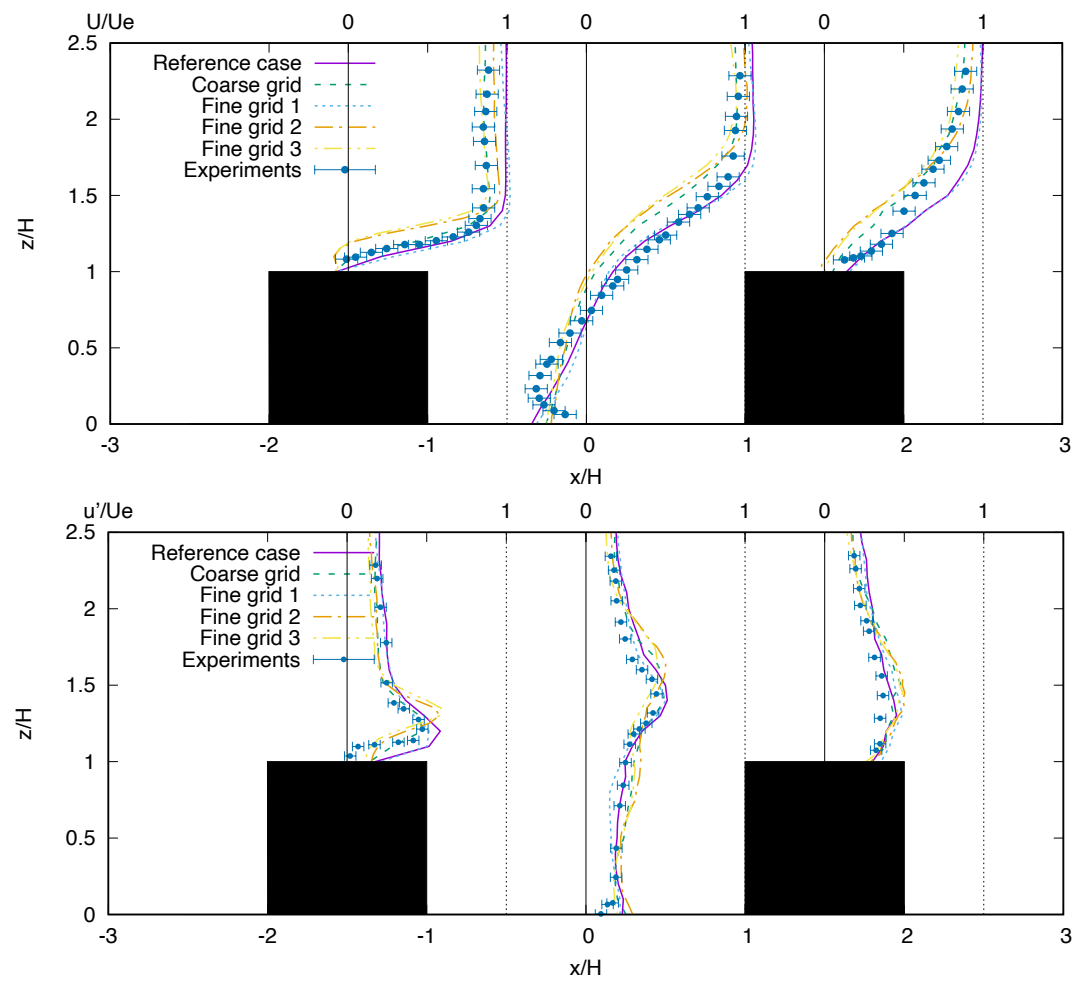

Figure A1. Mean streamwise velocity (top) and RMS streamwise velocity fluctuation $u^{\prime}$ (down) for the $2 \mathrm{H}$ street canyon opening. Points - experiments. Lines - reference case. Dashed lines - tests.

As shown on Figure A1, no notable differences have been observed in the mean fluid velocity and $u^{\prime}$ profiles when grid refinement is applied in the streamwise and spanwise directions (fine grid 1 case). Therefore, the difference in grid size in the spanwise and streamwise directions does not affect the simulation results.

When only $\Delta z_{\min }$ is decreased (fine grid 2), differences appear in the fluid velocity profiles (Figure A1). However, these discrepancies roughly fall within an acceptable deviation from the interval of experimental errors. The same is observed for fine grid 3 shown on Figure A1.

Particles were also added to the fine grid 2 case. Particle concentration and velocity profiles for the case of fine grid 2 are shown on Figure A2. As illustrated, vertical grid refinement has almost no impact on the observed solid particle concentration and velocity profiles.

\section{Acknowledgments}

We acknowledge NFSC/ANR chinese/french program PEDO-COTESOF. This work was granted access to the HPC resources of CINES. Numerical simulations were also performed on the P2CHPD parallel cluster. The model data used in this study (fluid and particle statistics) are available at the following repository: HydroShare, https://doi.org/10 $.4211 /$ hs . 148ef 9 f55909490fa442a4bde5e16251 

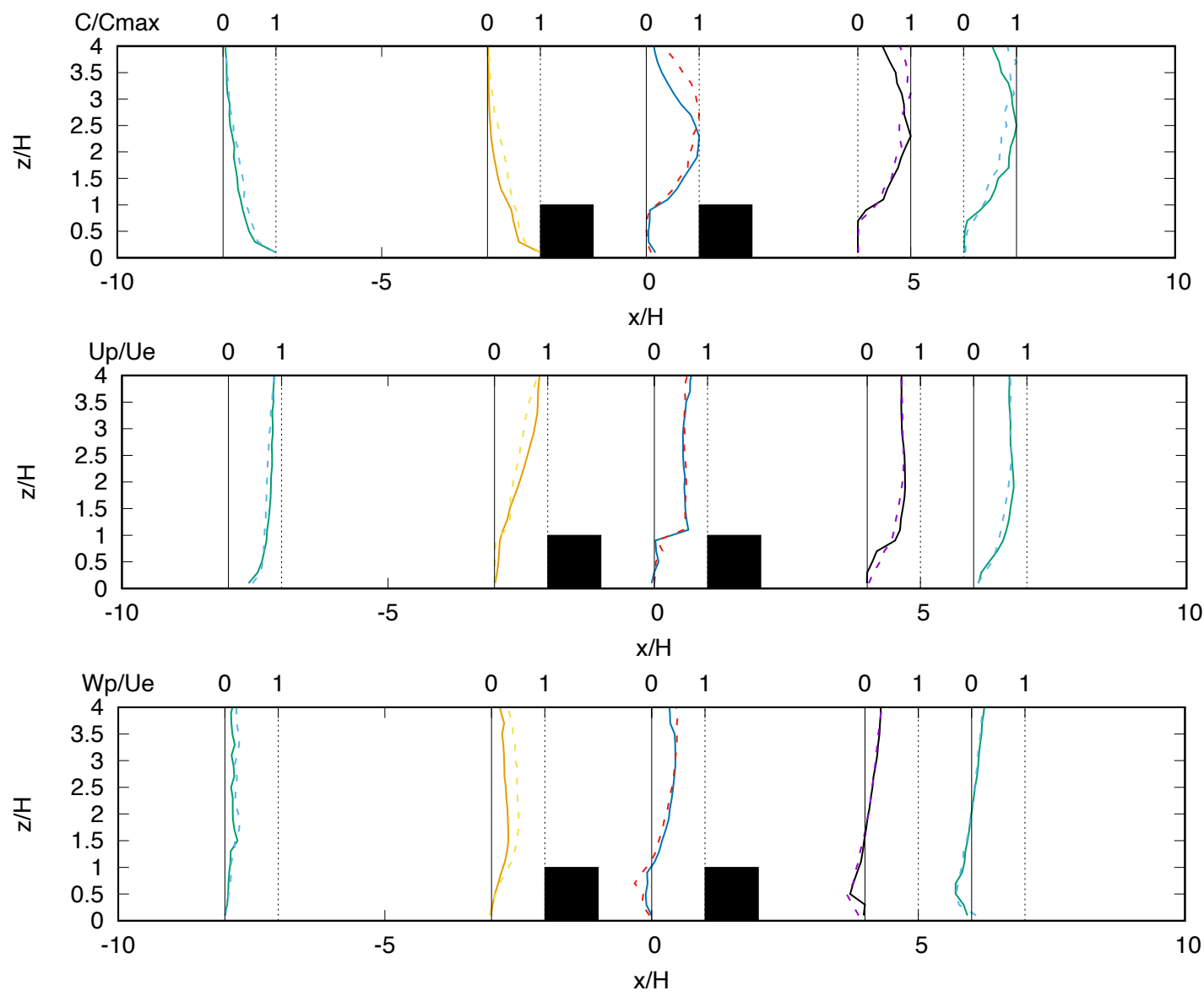

Figure A2. Fine grid 2 test with solid particles for the $2 H$ street canyon opening - mean concentration profiles (top), mean particle streamwise velocity profiles (middle), mean particle vertical velocity profiles (down). Lines - reference case. Dashed lines - fine grid 2 case. 


\section{References}

Anderson, R., \& Haff, P. (1991). Wind modification and bed response during saltation of sand in air. In Aeolian grain transport 1 (pp. 21-51). Springer

Araújo, A., Partelli, E., Pöschel, T., Andrade, J., \& Herrman, H. (2013). Numerical modeling of the wind over a tranverse dune. Nature Scientific Reports, 3, 2858.

Bagnold, R. A. (1941). The physics of blown sand and desert dunes. london: Methuen. Menthuen, London.

Beladjine, D., Ammi, M., Oger, L., \& Valance, A. (2007). Collision process between an incident bead and a three-dimensional granular packing. Physical Review E, $75(6), 061305$.

Castro, I., \& Robins, A. (1977). The flow around a surface-mouted cube in uniform and turbulent streams. Journal of Fluid Mechanics, 79, 307-335.

Casulli, V., \& Cheng, R. (1992). Semi-implicit finite difference methods for the three-dimensional shallow water flow. International Journal for Numerical in Fluids, 15, 629-648.

Clift, R., Grace, J., \& Weber, M. (1978). Bubbles, drops and particles. Academic Press, New York.

Creyssels, M., Dupont, P., El Moctar, A., Valance, A., Cantat, I., Jenkins, J., ... Rasmussen, K. (2009). Saltating particles in a turbulent boundary layer: experiment and theory. Journal of Fluid Mechanics, 625, 47-74.

Descamps, I., Harion, J., \& Baudoin, B. (2005). Taking-off model of particles with a wide size distribution. Chemical Engineering and Processing, 44, 159-166.

Diplat, P., \& Dancey, C. (2013). Coherent flow structures, initiation of motion, sediment transport and morphological feedbacks in rivers. Coherent Structures at Earth's surface, 44, 289-307.

Dupont, S., Bergametti, G., Marticorena, B., \& Simoëns, S. (2013). Modeling saltation intermittency. Journal of Geophysical Research: Atmospheres, 118(13), 7109-7128.

Dupont, S., Bergametti, G., \& Simoëns, S. (2014). Modeling aeolian erosion in presence of vegetation. Journal of Geophysical Research: Earth Surface, 119, 168187.

Durán, O., Claudin, P., \& Andreotti, B. (2011). On aeolian transport: grain-scale interactions, dynamical mechanisms and scaling laws. Aeolian Research, 3, 243270 .

Elghobashi, S. (1994). On predicting particle-laden turbulent flows. Applied scientific research, 52(4), 309-329.

Escauriaza, C., \& Sotiropoulos, F. (2011a). Initial stages of erosion and bed form development in a turbulent flow around a cylindrical pier. Journal of Geophysical Research: Earth Surface, 116, 1-24.

Escauriaza, C., \& Sotiropoulos, F. (2011b). Lagrangian model of bed-load transport in turbulent junction flows. Journal of Fluid Mechanics, 666, 36-76.

Foucaut, J.-M., \& Stanislas, M. (1996). Take-off threshold velocity of solid particles lying under a turbulent boundary layer. Experiments in Fluids, 20(5), $377-382$.

Grigoriadis, D., \& Kassinos, S. (2009). Lagrangian particle dispersion in turbulent flow over a wall mounted obstacle. International Journal of Heat and Fluid Flow, 30, 462-470.

Grimmond, C., \& Oke, T. (1999). Aerodynamics properties of urban areas derived from analysis of surface form. Journal of Applied Meteorology, 38, 1262-1292.

Huang, G. (2015). Numerical simulation of solid particle transport in atmospheric boundary-layer over obstacles. (Doctoral dissertation https: / / tel . archives -ouvertes.fr/tel-01303749/document), Ecole Centrale de Lyon, France.

Huang, G., Le Ribault, C., Vinkovic, I., \& Simoëns, S. (2018). Part i: a priori study of erosion and deposition with large eddy simulation of turbulent flow over 
multiple 2d sandy gaussian hills. Environmental Fluid Mechanics, 1-29.

Huang, G., Le Ribault, C., Vinkovic, I., \& Simoëns, S. (2019). Large eddy simulation of erosion and deposition over multiple two-dimensional gaussian hills in a turbulent boundary layer. Boundary Layer Meteorology, 193-222.

Huang, G., Simoëns, S., Vinkovic, I., Le Ribault, C., Dupont, S., \& Bergametti, G. (2016). Law-of-the-wall in a boundary-layer over regularly distributed roughness elements. Journal of Turbulence, 17, 518541.

Huang, N., He, P., \& Zhang, J. (2020). Large-eddy simulation of sand transport under unsteady wind. Geomorphology, 358(107105).

Kawamura, K. (1951). Study on sand movement by wind. Tokoy Daigaku Rikogaku Kenkyusho HOKOKU.

Klose, M., \& Shao, Y. (2013). Large eddy simulation of turbulent dust emission. Aeolian Research, 8, 49-58.

Kok, J., \& Renno, N. (2009). A comprehensive numerical model of steady state saltation (comsalt). Journal of Geophysical Research: Atmospheres, 114(D17).

Le Ribault, C., Vignon, J., \& Simoens, S. (2014a). Les/lagrangian modelling for the dispersion of reactive species behind an obstacle in a turbulent boundary layer. $J$ of Heat Mass Transfer, 9(1), 21-55.

Le Ribault, C., Vignon, J., \& Simoens, S. (2014b). Les/lagrangian modelling for the dispersion of reactive species behind an obstacle in a turbulent boundary layer. JP Journal of Heat and Mass Transfer, 9(1), 21-55.

Lu, P., Dong, Z., \& Ma, X. (2016). Aeolian sand transport above three desert surfaces in northern china with different characteristics (shifting sand, straw checker-board, and gravel): field observations. Environmental Earth Science, 75,577 .

Lund, T., Wu, X., \& Squires, K. (1998). Generation of turbulent inflow data for spatially-developing boundary layer simulations. Journal of Computational Physics, 140(2), 233-258.

Mittal, R., \& Iaccarino, G. (2005). Immersed boundary methods. Annual Review of Fluid Mechanics, 37, 239-261.

Oke, T. (1988). Street design and urban canopy layer climate. Energy and buildings, $11(1-3), 103-113$.

Sato, T., Hagishima, A., Ikegaya, N., \& Tanimoto, J. (2015). Wind tunnel experiment on turbulent flow field around $2 \mathrm{~d}$ street canyon with eaves. ICUC9-9th International Conference on Urban Climate.

Schmeeckle, M. (2014). Numerical simulation of turbulence and sediment transport of medium sand. Journal of Geophysical Research: Earth Surface, 119, 12401262 .

Schmeeckle, M., Nelson, J., \& Shreve, R. (2007). Forces on stationary particles in near-bed turbulent flows. Journal of Geophysical Research: Earth Surface, 112, F02003.

Shao, Y. (2008). Physics and modelling of wind erosion (Vol. 37). Springer Science \& Business Media.

Shao, Y., \& Leslie, L. (1997). Wind erosion prediction over australian continent. Journal of Geophysical Research, 1023, 30091-30106.

Shao, Y., \& Li, A. (1999). Numerical modelling of saltation in the atmospheric surface layer. Boundary-layer Meteorology, 91, 199-225.

Simoens, S., Ayrault, M., \& Wallace, J. (2007). The flow across a street canyon of variable width. part 1: Kinematic description. Atmospheric Environment, $41(39), 9002-9017$.

Simoëns, S., Saleh, A., Leribault, C., Belhmadi, M., Zegadi, R., Allag, F., ... Huang, G. (2015). Influence of gaussian hill on concentration of solid particles in suspension inside turbulent boudary layer. Procedia IUTAM, 17, 110-118.

Simoëns, S., \& Wallace, J. (2008). The flow across a street canyon of variable width- 
part 2: Scalar dispersion for the flow across a street canyon of variable width. Atmospheric Environment, 42(10), 2489-2503.

Sørensen, M. (1991). An analytic model of wind-blown sand transport. Acta Mechanica, 67-81.

Sumer, B., Chua, L., \& Cheng, N. (2003). Influence of turbulence on bed load sediment transport. Journal of Hydraulic Engineering, 129, 585-596.

Uehara, K., Wakamatsu, S., \& Ooka, R. (2003). Studies on critical reynolds number indices for wind-tunnel experiments on flow within urban areas. Boundary Layer Meteorology, 107(2), 353-370.

Uhlmann, M. (2005). An immersed boundary method with direct forcing for the simulation of particulate flows. Journal of Computational Physics, 209, 448476. doi: $10.1016 /$ j.jcp.2005.03.017

Vinçont, J.-Y., Simoens, S., Ayrault, M., \& Wallace, J. (2000). Passive scalar dispersion in a turbulent boundary layer from a line source at the wall and downstream of an obstacle. Journal of Fluid Mechanics, 424, 127-167.

Vinkovic, I., Aguirre, C., Ayrault, M., \& Simoëns, S. (2006). Large-eddy simulation of the dispersion of solid particles in a turbulent boundary layer. BoundaryLayer Meteorology, 121(2), 283.

Wang, P., Feng, S., Zheng, X., \& Sung, H. (2019). The scale characteristics and formation mechanism of aeolian sand streamers based on large eddy simulation. Journal of Geophysical Research: Atmospheres, 124(11), 372-388.

Wang, Z., \& Huang, N. (2017). Numerical simulation of the falling snow deposition over complex terrain. Journal of Geophysical Research: Atmospheres, 122, 980-1000.

$\mathrm{Wu}$, J. (2019). Numerical simulation of wind erosion : application to dune migration. (Doctoral dissertation https: //tel . archives-ouvertes $\cdot \mathrm{fr} / \mathrm{tel}$ -02166919/document), Ecole Centrale de Lyon, France.

Xu, B., Zhang, J., Huang, N., Gong, K., \& Liu, Y. (2018). Characteristics of turbulent aeolian sand movement over straw checkerboard barriers and formation mechanisms of their internal erosion form. Journal of geophysical research: Atmospheres.

Xue, M., Droegemeier, K., \& Wong, V. (2000). The advanced regional prediction system (arps)-a multi-scale nonhydrostatic atmospheric simulation and prediction model. part i: Model dynamics and verification. Meteorology and Atmospheric Physics, 75(3-4), 161-193.

Xue, M., Droegemeier, K., Wong, V., Shapiro, A., \& Brewster, K. (1995). Advanced regional prediction system (arps) version 4.0 user's guide. Center for Analysis and Prediction of Storms, University of Oklahoma, 380.

Xue, M., Droegemeier, K., Wong, V., Shapiro, A., Brewster, K., Carr, F., ... Wang, D. (2001). The advanced regional prediction system (arps)-a multi-scale nonhydrostatic atmospheric simulation and prediction tool. part ii: Model physics and applications. Meteorology and Atmospheric Physics, 76(3-4), 143-165.

Yamamoto, Y., Potthoff, M., Tanaka, T., T. Kashijima, \& Tsuji, Y. (2001). Largeeddy simulation of turbulent gas-particle flow in a vertical channel: effects of considering inter-particle collisions. Journal of Fluid Mechanics, 442, 303334

Yoshizawa, A. (1982). A statistically-derived subgrid model for the large-eddy simulation of turbulence. Physics of Fluids, 25(9), 1532-1538. 